\title{
Adaptive Morley element algorithms for the biharmonic eigenvalue problem
}

Hao Li and Yidu Yang*

\section{"Correspondence:}

ydyang@gznu.edu.cn

The School of the Mathematical

Sciences, Guizhou Normal

University, Gui Yang, China

\section{Springer}

\begin{abstract}
This paper is devoted to the adaptive Morley element algorithms for a biharmonic eigenvalue problem in $\mathbb{R}^{n}(n \geq 2)$. We combine the Morley element method with the shifted-inverse iteration including Rayleigh quotient iteration and the inverse iteration with fixed shift to propose multigrid discretization schemes in an adaptive fashion. We establish an inequality on Rayleigh quotient and use it to prove the efficiency of the adaptive algorithms. Numerical experiments show that these algorithms are efficient and can get the optimal convergence rate.
\end{abstract}

MSC: $65 \mathrm{~N} 25 ; 65 \mathrm{~N} 30 ; 65 \mathrm{~N} 15$

Keywords: Biharmonic eigenvalues; Morley elements; Adaptive algorithms; An inequality on Rayleigh quotient

\section{Introduction}

Biharmonic equation/eigenvalue problem plays an important role in elastic mechanics. In 1968, Morley designed a famous non-conforming element called the Morley element [1] to solve biharmonic equation (plate bending problem). The Morley element was extended to arbitrarily dimensions by Wang and $\mathrm{Xu}$ [2] in 2006. For biharmonic equation, the a priori/a posteriori error estimate was studied in [3-6] and the convergence and optimality of the adaptive Morley element method was proved in $[7,8]$. The Morley element has been employed to solve the biharmonic eigenvalue problem, including the vibration of a plate; and [9] studied its a priori error estimate. [10,11] studied a posteriori error estimate and the adaptive method, [12] adopted a new method dispensing with any additional regularity assumption to study the error estimates and adaptive algorithms. This paper further studies the adaptive Morley element method and has the following features:

1. The adaptive finite element methods, which were first proposed by Babuska and Rheinboldt [13], have gained an extensive attention in academia. More and more researchers entered this field and obtained many good results, most of which have been systemically summarized in $[5,14-16]$. And $[10,12]$ have employed the adaptive Morley element algorithms for the biharmonic eigenvalue problem based on solving directly the original eigenvalue problem $a(u, v)=\lambda b(u, v)$ in each iteration. In this paper, we establish the adaptive Morley element algorithms based on the shifted-inverse iteration including Rayleigh quotient iteration and the inverse iteration with fixed shift to solve the biharmonic eigenvalue problem. The 
shifted-inverse iteration method based on the multigrid discretizations has been studied in-depth (see [17] and the references therein), but they did not involve the Morley element. With our method, the solution of an original eigenvalue problem is reduced to the solution of an eigenvalue problem on a much coarser grid and the solution of a series of linear algebraic equations on finer and finer grids. Therefore, our method is more efficient than the method in $[10,12]$.

2. For fourth order equations in $\mathbb{R}^{3}$, it is difficult to employ a conforming element. For instance, Zenicek constructed a conforming tetrahedral finite element with 9 degree of polynomials and 220 nodal parameters [5], while the Morley tetrahedral element [2] has only 10 nodal parameters. Based on [4], we comply with the adaptive Morley element computation for the biharmonic eigenvalue problem in $\mathbb{R}^{3}$. Numerical results indicate that the adaptive algorithms are very efficient.

3. A family of good adaptive meshes should satisfy $h=O\left(h_{\min }^{\alpha}\right)$, where $h$ is the mesh size, $h_{\min }$ is the diameter of the smallest element, and $\alpha$ is the regularity index of the biharmonic equation over the domain with reentrant corner (see [18]). However, we find through the numerical computation that $\frac{h}{h_{\min }^{\alpha}}$ will become bigger and bigger when the iteration increases for the standard adaptive algorithm. Thus, referring to [19], we combine the standard local refined adaptive algorithm with uniformly refined algorithm to give new algorithms.

\section{Preliminary}

Consider the following biharmonic eigenvalue problem:

$$
\begin{aligned}
& \Delta^{2} u=\lambda u, \quad \text { in } \Omega, \\
& \frac{\partial u}{\partial \gamma}=0, \quad u=0, \text { on } \partial \Omega,
\end{aligned}
$$

where $\Omega \in \mathbb{R}^{n}$ is a polyhedral domain with boundary $\partial \Omega, \frac{\partial u}{\partial \gamma}$ is the outward normal derivative on $\partial \Omega$.

Let $H^{s}(\Omega)$ denote a usual Sobolev space with norm $\|\cdot\|_{s, \Omega}\left(\|\cdot\|_{s}\right), H_{0}^{2}(\Omega)=\left\{v \in H^{2}(\Omega)\right.$ : $\left.\left.v\right|_{\partial \Omega}=\left.\frac{\partial v}{\partial \gamma}\right|_{\partial \Omega}=0\right\}$ with norm $\|\cdot\|_{2}$ and semi-norm $|\cdot|_{2}$.

The weak form of $(2.1)$ is to seek $(\lambda, u) \in \mathbb{R} \times H_{0}^{2}(\Omega)$ with $u \neq 0$ such that

$$
a(u, v)=\lambda b(u, v), \quad \forall v \in H_{0}^{2}(\Omega)
$$

where

$$
a(u, v)=\int_{\Omega} \sum_{1 \leq i, j \leq n} \frac{\partial^{2} u}{\partial x_{i} \partial x_{j}} \frac{\partial^{2} v}{\partial x_{i} \partial x_{j}} d x, \quad b(u, v)=\int_{\Omega} u v d x, \quad\|u\|_{b}=\sqrt{b(u, u)} .
$$

In the case of $n=2,(2.2)$ is the weak form of clamped plate vibration.

It is easy to verify that $a(u, v)$ is a symmetric, continuous, and $H_{0}^{2}(\Omega)$-elliptic bilinear form. Let $\|u\|_{a}=\sqrt{a(u, u)}$, then the norms $\|u\|_{a},\|u\|_{2}$, and $|u|_{2}$ are equivalent.

We assume that $\pi_{h}=\{\kappa\}$ is a regular simplex partition of $\Omega$ and satisfies $\bar{\Omega}=\bigcup \bar{\kappa}$ (see [20]). Let $h_{\kappa}$ be the diameter of $\kappa$, and $h=\max \left\{h_{\kappa}: \kappa \in \pi_{h}\right\}$ be the mesh size of $\pi_{h}(h<1)$, $h_{\min }=\min \left\{h_{\kappa}: \kappa \in \pi_{h}\right\}$. Let $\varepsilon_{h}=\{F\}$ denote the set of faces $\left((n-1)\right.$-simplexes) of $\pi_{h}$, and 
let $\varepsilon_{h}^{\prime}=\{l\}$ denote the set of faces $(n-2)$-simplexes of $\pi_{h}$. When $n=2, l=z$ is a vertex of $\kappa$, and $\frac{1}{\text { meas }(l)} \int_{l} v=v(z)$. Let $\pi_{h}(\kappa)$ denote the set of all elements sharing a common face with the element $\kappa$. Let $\kappa_{+}$and $\kappa_{-}$be any two $n$-simplexes with a face $F$ in common such that the unit outward normal to $\kappa_{-}$at $F$ corresponds to $\gamma_{F}$. We denote the jump of $v$ across the face $F$ by

$$
[v]=\left.\left(\left.v\right|_{\kappa_{+}}-\left.v\right|_{\kappa_{-}}\right)\right|_{F}
$$

And the jump on boundary faces is simply given by the trace of the function on each face. In the papers [2, 5], the Morley element space is defined by

$$
\begin{aligned}
S^{h}= & \left\{v \in L_{2}(\Omega):\left.v\right|_{\kappa} \in P_{2}(\kappa), \forall \kappa \in \pi_{h}, \int_{F}\left[\nabla v \cdot \gamma_{F}\right]=0 \forall F \in \varepsilon_{h},\right. \\
& \left.\frac{1}{\operatorname{meas}(l)} \int_{l}[v]=0 \forall l \in \varepsilon_{h}^{\prime}\right\},
\end{aligned}
$$

where $P_{2}(\kappa)$ denotes the space of polynomials of degree less than or equal to 2 on $\kappa$.

Define the interpolation operator $I_{h}: H_{0}^{2}(\Omega) \rightarrow S^{h}$, which satisfies

$$
\int_{F} \frac{\partial I_{h} v}{\partial \gamma}=\int_{F} \frac{\partial v}{\partial \gamma} \quad \forall F \in \varepsilon_{h}, \quad \frac{1}{l} \int_{l} I_{h} v=\frac{1}{l} \int_{l} v \quad \forall l \in \varepsilon_{h}^{\prime} .
$$

The Morley element space $S^{h} \subset L_{2}(\Omega), S^{h} \not \subset H^{1}(\Omega)$. Let

$$
\|v\|_{m, h}^{2}=\sum_{\kappa \in \pi_{h}}\|v\|_{m, \kappa}^{2}, \quad|v|_{m, h}^{2}=\sum_{\kappa \in \pi_{h}}|v|_{m, \kappa}^{2}, \quad m=0,1,2
$$

From Lemma 8 in [2], we know that $|\cdot|_{2, h}$ is equivalent to $\|\cdot\|_{2, h},\|\cdot\|_{2, h}$ is a norm in $S^{h}$, and $a_{h}(\cdot, \cdot)$ is a uniformly $S^{h}$-elliptic bilinear form, and $\|\cdot\|_{h}=a_{h}(\cdot, \cdot)^{\frac{1}{2}}$ is a norm in $S^{h}$. And the following equality holds for any $w \in H_{0}^{2}(\Omega)$ :

$$
\lim _{h \rightarrow 0} \inf _{v \in S^{h}}\|w-v\|_{h}=0
$$

The discrete form of (2.2) reads: Find $\left(\lambda_{h}, u_{h}\right) \in \mathbb{R} \times S^{h}$ with $u_{h} \neq 0$ such that

$$
a_{h}\left(u_{h}, v\right)=\lambda_{h} b\left(u_{h}, v\right), \quad \forall v \in S^{h},
$$

where

$$
a_{h}\left(u_{h}, v\right)=\sum_{\kappa \in \pi_{h}} \int_{\kappa} \sum_{i, j=1}^{n} \frac{\partial^{2} u_{h}}{\partial x_{i} \partial x_{j}} \frac{\partial^{2} v}{\partial x_{i} \partial x_{j}} d x .
$$

The corresponding boundary value problem of (2.1) is

$$
\begin{aligned}
& \Delta^{2} w=f, \quad \text { in } \Omega, \\
& \frac{\partial w}{\partial \gamma}=0, \quad w=0, \text { on } \partial \Omega .
\end{aligned}
$$


From [18], we know that

$$
\|w\|_{2+\alpha} \lesssim\|f\|_{0}
$$

where $\alpha \in\left(\frac{1}{2}, 1\right)$ for the domain with reentrant corner, and $\alpha=1$ for the convex domain in $\mathbb{R}^{2}$.

The weak form of (2.4) and its discrete form are to find $w \in H_{0}^{2}(\Omega)$ such that

$$
a(w, v)=b(f, v), \quad \forall v \in H_{0}^{2}(\Omega)
$$

and to find $w_{h} \in S^{h}$ such that

$$
a_{h}\left(w_{h}, v\right)=b(f, v), \quad \forall v \in S^{h} .
$$

Define the solution operators $T: L_{2}(\Omega) \rightarrow H_{0}^{2}(\Omega) \subset L_{2}(\Omega)$ and $T_{h}: L_{2}(\Omega) \rightarrow S^{h}$ as follows:

$$
\begin{aligned}
& a(T f, v)=b(f, v), \quad \forall v \in H_{0}^{2}(\Omega), \\
& a_{h}\left(T_{h} f, v\right)=b(f, v), \quad \forall v \in S^{h} .
\end{aligned}
$$

Then $T, T_{h}: L_{2}(\Omega) \rightarrow L_{2}(\Omega)$ are self-adjoint and compact.

It is well known that the eigenvalue problem (2.1) has countably many eigenvalues, which are real and positive diverging to $+\infty$. Suppose that $\lambda$ and $\lambda_{h}$ are the $k$ th eigenvalue of (2.2) and (2.3), respectively, the algebraic multiplicity of $\lambda$ is equal to $q, \lambda=\lambda_{k}=$ $\lambda_{k+1}=\cdots=\lambda_{k+q-1}$. Let $M(\lambda)$ be the space spanned by all eigenfunctions corresponding to $\lambda$ and $M_{h}(\lambda)$ be the direct sum of eigenspaces corresponding to all eigenvalues of (2.3) that converge to $\lambda$. Let $\hat{M}(\lambda)=\left\{u: u \in M(\lambda),\|u\|_{h}=1\right\}$.

Now we introduce the following quantity:

$$
\delta_{h}(\lambda)=\left\|\left.\left(T-T_{h}\right)\right|_{M\left(\lambda_{k}\right)}\right\|_{h} .
$$

The saturation condition was analyzed in [21-23], especially, it was analyzed in [22] for very general cases. According to this condition, we can make the following assumption:

$$
C_{1} h \leq \inf _{\forall v \in S^{h}}\|u-v\|_{h} \leq C_{2} \delta_{h}\left(\lambda_{k}\right), \quad \forall u \in M\left(\lambda_{k}\right)
$$

where $C_{1}$ and $C_{2}$ are independent of mesh parameters.

Define $S^{h}+H_{0}^{2}(\Omega)=\left\{v_{h}+v: v_{h} \in S^{h}, v \in H_{0}^{2}(\Omega)\right\}$.

Due to the generalized Poincare-Friedrichs inequality, Theorem 3 in [24] and $a_{h}(u-$ $\left.I_{h} u, v\right)=0, \forall v \in S^{h}$ (see [5]), we deduce for any $w \in S^{h}, u \in H_{0}^{2}(\Omega)$

$$
\begin{aligned}
\|w-u\|_{0} & \leq\left\|w-I_{h} u\right\|_{0}+\left\|u-I_{h} u\right\|_{0} \\
& \leq \frac{C_{3}}{3}\left(\left\|w-I_{h} u\right\|_{h}+h^{2}\left\|u-I_{h} u\right\|_{h}\right) \\
& \leq \frac{C_{3}}{3}\left(\|w-u\|_{h}+\left\|u-I_{h} u\right\|_{h}+h^{2}\left\|u-I_{h} u\right\|_{h}\right) \leq C_{3}\|w-u\|_{h} .
\end{aligned}
$$


Therefore,

$$
\|v\|_{0} \leq C_{3}\|v\|_{h}, \quad \forall v \in S^{h}+H_{0}^{2}(\Omega)
$$

where $C_{3}$ is a positive constant independent of mesh parameters.

From (2.5) we have the following estimate using the Cauchy-Schwarz inequality: For any $g \in L^{2}(\Omega), T_{h} g \in S^{h}$ satisfying

$$
\left\|T_{h} g\right\|_{h} \leq C_{3}\|g\|_{0}
$$

define the consistency term

$$
E_{h}\left(w, v_{h}\right)=a_{h}(w, v)-b(f, v), \quad \forall v \in S^{h}+H_{0}^{2}(\Omega) .
$$

Suppose $w \in H^{2+r}(\Omega), r \in\left(\frac{1}{2}, 1\right]$, then we have the following estimate:

$$
\left|E_{h}(w, v)\right| \leq C_{4} h^{r}\left(\|w\|_{2+r}+h^{2-r}\|f\|_{0}\right)\|v\|_{h}, \quad \forall v \in S^{h}+H_{0}^{2}(\Omega) .
$$

Using the trace inequality [5] proves the above estimate under the case $r=1$. Using the arguments in [5], we can obtain the above estimate under the case $r=\left(\frac{1}{2}, 1\right]$ (also see [11]). We can derive the following Lemma 2.1 from Lemma 2.3 in [25].

Lemma 2.1 Let $\lambda$ and $\lambda_{h}$ be the kth eigenvalue of (2.2) and (2.3), respectively. Then for any eigenfunction $u_{h}$ corresponding to $\lambda_{h}$ with $\left\|u_{h}\right\|_{h}=1$, there exist $u \in M(\lambda)$ and $h_{0}>0$ such that if $h \leq h_{0}$,

$$
\left\|u-u_{h}\right\|_{h} \leq C_{5} \delta_{h}(\lambda)
$$

for any $u \in \widehat{M}(\lambda)$, there exists $u_{h} \in M_{h}(\lambda)$ such that if $h \leq h_{0}$,

$$
\left\|u-u_{h}\right\|_{h} \leq C_{6} \delta_{h}(\lambda)
$$

where constants $C_{5}$ and $C_{6}$ are positive and only depend on $\lambda$.

The following inequality on Rayleigh quotient plays an important role.

Theorem 2.1 Let $(\lambda, u)$ be an eigenpair of (2.2), $v \in S^{h}$ with $\|v\|_{h}=1$ and $\|v-u\|_{h} \leq$ $\left(4 C_{3} \sqrt{\lambda}\right)^{-1}$, then the Rayleigh quotient $R(v)=\frac{a_{h}(v, v)}{\|v\|_{0}^{2}}$ satisfies

$$
|R(v)-\lambda| \leq C_{7}\|v-u\|_{h}^{1+r},
$$

where $C_{7}=4 \lambda\left(1+\lambda C_{3}^{2}\right)\left(4 C_{3} \sqrt{\lambda}\right)^{r-1}+\frac{8 C_{4}}{C_{1}^{r}} \lambda\left(\|u\|_{2+r}+h^{2-r} \lambda\|u\|_{0}\right)$.

Proof Since $u \in M(\lambda), v \in S^{h},\|v\|_{h}=1$ and $\|v-u\|_{h} \leq\left(4 C_{3} \sqrt{\lambda}\right)^{-1}$, by Lemma 3.1 in [26] we have

$$
\left\|v-\frac{u}{\|u\|_{h}}\right\|_{h} \leq 2\|v-u\|_{h} \leq\left(2 C_{3} \sqrt{\lambda}\right)^{-1}
$$




$$
\left\|v-\frac{u}{\|u\|_{h}}\right\|_{0} \leq C_{3}\left\|v-\frac{u}{\|u\|_{h}}\right\|_{h} \leq \frac{1}{2 \sqrt{\lambda}}
$$

which together with $\left\|\frac{u}{\|u\|_{h}}\right\|_{0}=\frac{1}{\sqrt{\lambda}}$ yields

$$
\|v\|_{0} \geq\left\|\frac{u}{\|u\|_{h}}\right\|_{0}-\left\|v-\frac{u}{\|u\|_{h}}\right\|_{0} \geq \frac{1}{2 \sqrt{\lambda}} .
$$

By Lemma 2.5 in [26], we get

$$
\frac{a_{h}(v, v)}{\|v\|_{0}^{2}}-\lambda=\frac{\|v-u\|_{h}^{2}}{\|v\|_{0}^{2}}-\lambda \frac{\|v-u\|_{0}^{2}}{\|v\|_{0}^{2}}+2 \frac{E_{h}(u, v)}{\|v\|_{0}^{2}} \text {. }
$$

Hence, from inequalities (2.7)-(2.9) we deduce

$$
\begin{aligned}
|R(v)-\lambda| & \leq 4 \lambda\|v-u\|_{h}^{2}+4 \lambda^{2}\|v-u\|_{0}^{2}+8 \lambda E_{h}(u, v) \\
& \leq 4 \lambda\|v-u\|_{h}^{2}+4 C_{3}^{2} \lambda^{2}\|v-u\|_{h}^{2}+8 \lambda E_{h}(u, v-u) \\
& \leq 4 \lambda\left(1+\lambda C_{3}^{2}\right)\|v-u\|_{h}^{2}+8 C_{4} h^{r} \lambda\left(\|u\|_{2+r}+h^{2-r} \lambda\|u\|_{0}\right)\|v-u\|_{h} \\
& \leq 4 \lambda\left(1+\lambda C_{3}^{2}\right)\|v-u\|_{h}^{2}+8 C_{4} \lambda\left(\|u\|_{2+r}+h^{2-r} \lambda\|u\|_{0}\right)\|v-u\|_{h}^{1+r} \\
& \leq\left(4 \lambda\left(1+\lambda C_{3}^{2}\right)\|v-u\|_{h}^{1-r}+\frac{8 C_{4}}{C_{1}^{r}} \lambda\left(\|u\|_{2+r}+h^{2-r} \lambda\|u\|_{0}\right)\right)\|v-u\|_{h}^{1+r} \\
& \leq\left(4 \lambda\left(1+\lambda C_{3}^{2}\right)\left(4 C_{3} \sqrt{\lambda}\right)^{r-1}+\frac{8 C_{4}}{C_{1}^{r}} \lambda\left(\|u\|_{2+r}+h^{2-r} \lambda\|u\|_{0}\right)\right)\|v-u\|_{h}^{1+r} \\
& \leq C_{7}\|v-u\|_{h}^{1+r} .
\end{aligned}
$$

We get the results that we need.

(2.3) implies $\lambda_{h}=R\left(u_{h}\right)$, and from (2.7), (2.10), and (3.22) in [11], we deduce

$$
\left|R\left(u_{h}\right)-\lambda\right| \leq C_{7}\left\|u_{h}-u\right\|_{h}^{2} \leq C_{5}^{2} C_{7} \delta_{h}^{2}(\lambda)
$$

\section{The shifted-inverse iteration based on multigrid discretization}

Let $\left\{S^{h_{i}}\right\}_{0}^{\infty}$ be a family Morley element spaces, $h_{0}=H$. Refer to the references [17], we present the following calculation schemes.

Scheme 1 (Rayleigh quotient iteration based on multigrid discretizations) Given the iteration times $l$.

Step 1. Solve (2.3) on $S^{H}$ : Find $\left(\lambda_{H}, u_{H}\right) \in \mathbb{R} \times S^{H}$ such that $\left\|u_{H}\right\|_{H}=1$ and

$$
a_{H}\left(u_{H}, v\right)=\lambda_{H} b\left(u_{H}, v\right), \quad \forall v \in S^{H} .
$$

Step 2. $u^{h_{0}} \Leftarrow u_{H}, \lambda^{h_{0}} \Leftarrow \lambda_{H}, i \Leftarrow 1$.

Step 3. Solve a linear system on $S^{h_{i}}$ : Find $u^{\prime} \in S^{h_{i}}$ such that

$$
a_{h}\left(u^{\prime}, v\right)-\lambda^{h_{i-1}} b\left(u^{\prime}, v\right)=b\left(u^{h_{i-1}}, v\right), \quad \forall v \in S^{h_{i}},
$$

$\operatorname{set} u^{h_{i}}=\frac{u^{\prime}}{\left\|u^{\prime}\right\|_{h}}$. 
Step 4. Compute the Rayleigh quotient:

$$
\lambda^{h_{i}}=\frac{a_{h}\left(u^{h_{i}}, u^{h_{i}}\right)}{b\left(u^{h_{i}}, u^{h_{i}}\right)}
$$

Step 5. If $i=l$, then output $\left(\lambda^{h_{l}}, u^{h_{l}}\right)$, stop; else, $i \Leftarrow i+1$, and return to Step 3 .

Scheme 2 (The inverse iteration with fixed shift based on multigrid discretizations) Given the iteration times $l$ and $i_{0}$.

Steps 1 4. The same as Steps 1-4 in Scheme 1.

Step 5. If $i>i_{0}$, then $\lambda^{h_{i_{0}}} \Leftarrow \lambda^{h_{i-1}}, i \Leftarrow i+1$, turn to Step 6; else, $i \Leftarrow i+1$, and return to Step 3.

Step 6. Solve a linear system on $S^{h_{i}}$ : Find $u^{\prime} \in S^{h_{i}}$ such that

$$
a_{h}\left(u^{\prime}, v\right)-\lambda^{h_{i_{0}}} b\left(u^{\prime}, v\right)=b\left(u^{h_{i-1}}, v\right), \quad \forall v \in S^{h_{i}},
$$

set $u^{h_{i}}=\frac{u^{\prime}}{\left\|u^{\prime}\right\|_{h}}$.

Step 7. Compute the Rayleigh quotient

$$
\lambda^{h_{i}}=\frac{a_{h}\left(u^{h_{i}}, u^{h_{i}}\right)}{b\left(u^{h_{i}}, u^{h_{i}}\right)} .
$$

Step 8. If $i=l$, then output $\left(\lambda^{h_{l}}, u^{h_{l}}\right)$, stop; else, $i \Leftarrow i+1$, and return to Step 6 .

Strictly speaking, the above $a_{h}(\cdot, \cdot)$ and $\|\cdot\|_{h}$ should be written as $a_{h_{i}}(\cdot, \cdot)$ and $\|\cdot\|_{h_{i}}$. For the sake of simplicity, we write $a_{h_{i}}(\cdot, \cdot)$ and $\|\cdot\|_{h_{i}}$ as $a_{h}(\cdot, \cdot)$ and $\|\cdot\|_{h}$, in this paper.

\section{The theoretical analysis}

In this section, we will prove the convergence of $\left(\lambda^{h_{l}}, u^{h_{l}}\right)$ derived from Scheme 1/ Scheme 2, and that the constants appearing in the error estimates are not only independent of mesh parameter but also iterative times $l$.

In the following discussion, let $\left(\lambda_{k}, u_{k}\right)$ and $\left(\lambda_{k, h}, u_{k, h}\right)$ denote the $k$ th eigenpair of (2.2) and (2.3), respectively, and $\mu_{k}=\frac{1}{\lambda_{k}}, \mu_{k, h}=\frac{1}{\lambda_{k, h}}, M\left(\mu_{k}\right)=M\left(\lambda_{k}\right), M_{h}\left(\mu_{k}\right)=M_{h}\left(\lambda_{k}\right)$.

Denote $\operatorname{dist}(u, S)=\inf _{v \in S}\|u-v\|_{h}$.

Our analysis is based on the following Lemma 4.1 (see Lemma 4.1 in [17]).

Lemma 4.1 Let $\left(\mu_{0}, u_{0}\right)$ be an approximation for $\left(\mu_{k}, u_{k}\right)$, where $\mu_{0}$ is not an eigenvalue of $T_{h}$, and $u_{0} \in S^{h}$ with $\left\|u_{0}\right\|_{h}=1$. Suppose that

(C1) $\operatorname{dist}\left(u_{0}, M_{h}\left(\mu_{k}\right)\right) \leq \frac{1}{2}$

(C2) $\left|\mu_{0}-\mu_{k}\right| \leq \frac{\rho}{4},\left|\mu_{j, h}-\mu_{j}\right| \leq \frac{\rho}{4}$ for $j=k-1, k, k+q(j \neq 0)$, where $\rho=\min _{\mu_{j} \neq \mu_{k}}\left|\mu_{j}-\mu_{k}\right|$ is the separation constant of the eigenvalue $\mu_{k}$;

(C3) $u^{\prime} \in S^{h}, u_{k}^{h} \in S^{h}$ satisfy

$$
\left(\mu_{0}-T_{h}\right) u^{\prime}=u_{0}, \quad u_{k}^{h}=\frac{u^{\prime}}{\|u\|_{h}},
$$

then the following inequality holds:

$$
\operatorname{dist}\left(u_{k}^{h}, M_{h}\left(\mu_{k}\right)\right) \leq \frac{4}{\rho} \max _{k \leq j \leq k+q-1}\left|\mu_{0}-\mu_{j, h}\right| \operatorname{dist}\left(u_{0}, M_{h}\left(\mu_{k}\right)\right) .
$$


Next, we will use the proof method in [17] to analyze the error of Schemes 1-2.

Let $\delta_{0}$ be a positive constant satisfying the following inequalities:

$$
\begin{aligned}
& \max \left\{1, C_{5}\right\} \delta_{0} \leq \min \left\{\frac{1}{2}, \frac{1}{4 C_{3} \sqrt{\lambda_{k}}}\right\} ; \\
& 4 C_{3} C_{7} \delta_{0}^{2}+4 C_{3}^{2} \lambda_{k} \delta_{0}+2 \lambda_{k} \delta_{0}+C_{6} \delta_{0} \leq \frac{1}{2} ; \\
& \frac{\delta_{0}}{\left(\lambda_{k}-\delta_{0}\right) \lambda_{k}} \leq \frac{\rho}{4}, \quad \delta_{0} \leq \frac{\lambda_{k}}{2} ; \\
& \frac{C_{5}^{2} C_{7} \delta_{0}^{2}}{\lambda_{j}\left(\lambda_{j}-C_{5}^{2} C_{7} \delta_{0}^{2}\right)} \leq \frac{\rho}{4}, \quad j=k-1, k, \ldots, k+q, j \neq 0 .
\end{aligned}
$$

Condition 4.1 There exists $\bar{u} \in M\left(\lambda_{k}\right)$ such that

$$
\left\|u^{h_{l}}-\bar{u}\right\|_{h} \leq \delta_{0}, \quad\left|\lambda_{0}-\lambda_{k}\right| \leq \delta_{0}, \quad \delta_{h_{l}}\left(\lambda_{j}\right) \leq \delta_{0} \quad(j=k-1, k, k+1, j \neq 0),
$$

where $\lambda_{0}$ is an approximate eigenvalue of $\lambda_{k}, u^{h_{l}}$ is an approximate eigenfunction obtained by Scheme 1 or Scheme 2, and $\rho$ is the separation constant of the eigenvalue $\mu_{k}=\frac{1}{\lambda_{k}}$.

Condition 4.1 plays a key role in proving Theorem 4.1, by which we can prove Theorems 4.2-4.3. In the proof of Theorems 4.2-4.3, we can deduce that Condition 4.1 holds when the mesh size $H$ is appropriately small. However, it is difficult to verify the condition whether the mesh size $H$ is appropriately small or not. And it seems to be a necessary condition in many papers on the convergence and error estimates of the finite element method for eigenvalue problem. But numerical experiments in Sect. 6 present a satisfying practical performance for our algorithms, which shows that it is unnecessary for the mesh size $H$ to be appropriately small, even though the theory is not complete.

The following Theorems 4.1-4.3 are the generalization of Theorems 4.2-4.4 in [17].

Theorem 4.1 Let $\left(\lambda_{k}^{h_{l}}, u_{k}^{h_{l}}\right)$ be an approximate eigenvalue obtained by Scheme 1 or Scheme 2. Assume that Lemma 2.1 and Condition 4.1 hold with $\lambda_{0}=\lambda_{k}^{h_{l-1}}$ for Scheme 1 or $\lambda_{0}=\lambda_{k}^{h_{i 0}}$ for Scheme 2 . Then there exists $u_{k} \in M\left(\lambda_{k}\right)$ such that

$$
\left\|u_{k}^{h_{l}}-u_{k}\right\|_{h} \leq \frac{C_{0}}{2}\left\{\left|\lambda_{0}-\lambda_{k}\right|\left(\left|\lambda_{k}^{h_{l-1}}-\lambda_{k}\right|+\left\|u_{k}^{h_{l-1}}-\bar{u}\right\|_{h}\right)+\delta_{h_{l}}\left(\lambda_{k}\right)\right\} .
$$

Proof We use Lemma 4.1 to complete the proof. Select $\mu_{0}=\frac{1}{\lambda_{0}}$ and $u_{0}=\frac{\lambda_{k}^{h_{l-1}} T_{h_{l}} u_{k}^{h_{l-1}}}{\left\|\lambda_{k}^{h_{l-1}} T_{h_{l}} u_{k}\right\|_{l-1} \|_{h}}$. Then, by (2.6) and (2.8), we have

$$
\begin{aligned}
& \left\|\lambda_{k}^{h_{l-1}} T_{h_{l}} u_{k}^{h_{l-1}}-\bar{u}\right\|_{h} \\
& \quad=\left\|\lambda_{k}^{h_{l-1}} T_{h_{l}} u_{k}^{h_{l-1}}-\lambda_{k} T_{h_{l}} u_{k}^{h_{l-1}}+\lambda_{k} T_{h_{l}} u_{k}^{h_{l-1}}-\lambda_{k} T_{h_{l}} \bar{u}+\lambda_{k} T_{h_{l}} \bar{u}-\lambda_{k} T \bar{u}\right\|_{h} \\
& \quad \leq C_{3}\left|\lambda_{k}^{h_{l-1}}-\lambda_{k}\right|+C_{3} \lambda_{k}\left\|u_{k}^{h_{l-1}}-\bar{u}\right\|_{0}+\lambda_{k}\left\|\left.\left(T_{h_{l}}-T\right)\right|_{M\left(\lambda_{k}\right)}\right\|_{h}\|\bar{u}\|_{h} \\
& \quad \leq C_{3}\left|\lambda_{k}^{h_{l-1}}-\lambda_{k}\right|+C_{3}^{2} \lambda_{k}\left\|u_{k}^{h_{l-1}}-\bar{u}\right\|_{h}+\lambda_{k} \delta_{h_{l}}\left(\lambda_{k}\right)\|\bar{u}\|_{h} .
\end{aligned}
$$


Noting that $\|\bar{u}\|_{h} \geq\left\|u_{k}^{h_{l-1}}\right\|_{h}-\left\|\bar{u}-u_{k}^{h_{l-1}}\right\|_{h} \geq 1-\delta_{0} \geq \frac{1}{2}$, thus, by Lemma 3.1 in [26], we have

$$
\begin{aligned}
\left\|u_{0}-\frac{\bar{u}}{\|\bar{u}\|_{h}}\right\|_{h} & \leq \frac{2}{\|\bar{u}\|_{h}}\left\|\lambda_{k}^{h_{l-1}} T_{h_{l}} u_{k}^{h_{l-1}}-\bar{u}\right\|_{h} \\
& \leq 4 C_{3}\left|\lambda_{k}^{h_{l-1}}-\lambda_{k}\right|+4 C_{3}^{2} \lambda_{k}\left\|u_{k}^{h_{l-1}}-\bar{u}\right\|_{h}+2 \lambda_{k} \delta_{h_{l}}\left(\lambda_{k}\right) .
\end{aligned}
$$

Using the triangle inequality, (4.7), (2.11), Condition 4.1 and (4.3), we get

$$
\begin{aligned}
& \operatorname{dist}\left(u_{0}, M_{h_{l}}\left(\lambda_{k}\right)\right) \\
& \quad \leq\left\|u_{0}-\frac{\bar{u}}{\|\bar{u}\|_{h}}\right\|_{h}+\operatorname{dist}\left(\frac{\bar{u}}{\|\bar{u}\|_{h}}, M_{h_{l}}\left(\lambda_{k}\right)\right) \\
& \quad \leq 4 C_{3}\left|\lambda_{k}^{h_{l-1}}-\lambda_{k}\right|+4 C_{3}^{2} \lambda_{k}\left\|u_{k}^{h_{l-1}}-\bar{u}\right\|_{h}+2 \lambda_{k} \delta_{h_{l}}\left(\lambda_{k}\right)+C_{6} \delta_{h_{l}}\left(\lambda_{k}\right) \\
& \quad \leq 4 C_{3} C_{7} \delta_{0}^{2}+4 C_{3}^{2} \lambda_{k} \delta_{0}+2 \lambda_{k} \delta_{0}+C_{6} \delta_{0} \leq \frac{1}{2} .
\end{aligned}
$$

From Condition 4.1, (4.4), we have

$$
\left|\mu_{0}-\mu_{k}\right|=\frac{\left|\lambda_{0}-\lambda_{k}\right|}{\lambda_{0} \lambda_{k}} \leq \frac{\delta_{0}}{\left(\lambda_{k}-\delta_{0}\right) \lambda_{k}} \leq \frac{\rho}{4}
$$

From (2.13), we deduce

$$
\left|\mu_{j}-\mu_{j, h_{l}}\right|=\left|\frac{\lambda_{j}-\lambda_{j, h_{l}}}{\lambda_{j} \lambda_{j, h_{l}}}\right| \leq \frac{C_{5}^{2} C_{7} \delta_{h}(\lambda)^{2}}{\lambda_{j}\left(\lambda_{j}-C_{5}^{2} C_{7} \delta_{h}(\lambda)^{2}\right)} \leq \frac{C_{5}^{2} C_{7} \delta_{0}^{2}}{\lambda_{j}\left(\lambda_{j}-C_{5}^{2} C_{7} \delta_{0}^{2}\right)} \leq \frac{\rho}{4}
$$

Hence, the conditions in Lemma 4.1 are verified.

By (2.5) we see that Step 3 in Scheme 1 or Step 6 in Scheme 2 is equivalent to the following:

$$
\begin{aligned}
& a_{h}\left(u^{\prime}, v\right)-\lambda_{0} a_{h}\left(T_{h_{l}} u^{\prime}, v\right)=a_{h}\left(T_{h_{l}} u_{k}^{h_{l-1}}, v\right), \quad \forall v \in S^{h_{l}}, \\
u_{k}^{h_{l}}= & \frac{u^{\prime}}{\left\|u^{\prime}\right\|_{h}}, \text { i.e., } \\
& \left(\lambda_{0}^{-1}-T_{h_{l}}\right) u^{\prime}=\lambda_{0}^{-1} T_{h_{l}} u_{k}^{h_{l-1}}, \quad u_{k}^{h_{l}}=\frac{u^{\prime}}{\left\|u^{\prime}\right\|_{h}} .
\end{aligned}
$$

Then Step 3 in Scheme 1 or Step 6 in Scheme 2 is equivalent to

$$
\left(\lambda_{0}^{-1}-T_{h_{l}}\right) u^{\prime}=u_{0}, \quad u_{k}^{h_{l}}=\frac{u^{\prime}}{\left\|u^{\prime}\right\|_{h}}
$$

From (4.4), (2.13) and (4.5), we derive that

$$
\begin{aligned}
\left|\mu_{0}-\mu_{j, h_{l}}\right| & =\left|\frac{1}{\lambda_{0}}-\frac{1}{\lambda_{j, h_{l}}}\right| \leq \frac{4\left|\lambda_{0}-\lambda_{j, h_{l}}\right|}{\lambda_{k}^{2}} \leq \frac{4}{\lambda_{k}^{2}}\left|\lambda_{0}-\lambda_{k}\right|+\frac{4}{\lambda_{k}^{2}}\left|\lambda_{k}-\lambda_{j, h_{l}}\right| \\
& \leq \frac{4}{\lambda_{k}^{2}} \delta_{0}+\frac{4 C_{5}^{2} C_{7}}{\lambda_{k}^{2}} \delta_{0}^{2}, \quad j=k, k+1, \ldots, k+q-1 .
\end{aligned}
$$


Let the eigenvectors $\left\{u_{j, h_{l}}\right\}_{k}^{k+q-1}$ be an orthogonal basis of $M_{h_{l}}\left(\lambda_{k}\right)$ with respect to $a_{h}(\cdot, \cdot)$. Denote

$$
u^{*}=\sum_{j=k}^{k+q-1} a_{h}\left(u_{k}^{h_{l}}, u_{j, h_{l}}\right) u_{j, h_{l}},
$$

then

$$
\left\|u_{k}^{h_{l}}-u^{*}\right\|_{h}=\operatorname{dist}\left(u_{k}^{h_{l}}, M_{h_{l}}\left(\lambda_{k}\right)\right)
$$

Hence, substituting (4.8) and (4.9) into (4.1), we obtain

$$
\begin{aligned}
\left\|u_{k}^{h_{l}}-u^{*}\right\|_{h}= & \operatorname{dist}\left(u_{k}^{h_{l}}, M_{h_{l}}\left(\lambda_{k}\right)\right) \\
\leq & \frac{4}{\rho}\left(\frac{4}{\lambda_{k}^{2}}\left|\lambda_{0}-\lambda_{k}\right|+\frac{4 C_{5}^{2} C_{7}}{\lambda_{k}^{2}} \delta_{h}^{2}(\lambda)\right) \\
& \times\left(4 C_{3}\left|\lambda_{k}^{h_{l-1}}-\lambda_{k}\right|+4 C_{3}^{2} \lambda_{k}\left\|u_{k}^{h_{l-1}}-\bar{u}\right\|_{h}\right. \\
& \left.+2 \lambda_{k} \delta_{h_{l}}\left(\lambda_{k}\right)+C_{6} \delta_{h_{l}}\left(\lambda_{k}\right)\right) .
\end{aligned}
$$

By Lemma 2.1, there exist eigenvectors $\left\{u_{j}^{0}\right\}_{k}^{k+q-1}$ making $u_{j, h_{l}}$ and $u_{j}^{0}$ satisfy (2.10). Let

$$
u_{k}=\sum_{j=k}^{k+q-1} a_{h}\left(u_{k}^{h_{l}}, u_{j, h_{l}}\right) u_{j}^{0},
$$

then $u_{k} \in M\left(\lambda_{k}\right)$.

Using (2.10), we deduce that

$$
\begin{aligned}
\left\|u_{k}-u^{*}\right\|_{h} & =\left\|\sum_{j=k}^{k+q-1} a_{h}\left(u_{k}^{h_{l}}, u_{j, h_{l}}\right)\left(u_{j}^{0}-u_{j, h_{l}}\right)\right\|_{h} \\
& \leq\left(\sum_{j=k}^{k+q-1}\left\|u_{j}^{0}-u_{j, h_{l}}\right\|_{h}^{2}\right)^{\frac{1}{2}} \leq\left(C_{5}^{2} \delta_{h_{l}}\left(\lambda_{j}\right)^{2}\right)^{\frac{1}{2}} \leq q^{\frac{1}{2}} C_{5} \delta_{h_{l}}\left(\lambda_{j}\right) .
\end{aligned}
$$

Noting that the constants $C_{3}, C_{5}, C_{6}, C_{7}$ and $\rho$ are independent of mesh parameters and iterative times $l$, and $\left\|u_{k}^{h_{l-1}}-\bar{u}\right\|_{h} \leq \delta_{0},\left|\lambda_{0}-\lambda_{k}\right| \leq \delta_{0}$ and $\delta_{h_{l}}\left(\lambda_{k}\right) \leq \delta_{0}$, by (4.10) and (4.2), we know that there exists a positive constant $C_{0}$ that is independent of mesh parameters and $l$ such that (4.6) holds. And we can have $C_{0} \geq C_{5}$.

We need the following two conditions (see Conditions 4.2 and 4.3 in [17]).

Condition 4.2 There exists $t_{i} \in(1,2](i=1,2, \ldots)$ such that $\delta_{h_{i}}\left(\lambda_{k}\right)=\delta_{h_{i-1}}^{t_{i}}\left(\lambda_{k}\right)$ and $\delta_{h_{i}}\left(\lambda_{k}\right) \rightarrow 0(i \rightarrow \infty)$.

Condition 4.2 is easily satisfied; for example, for smooth eigenfunction, by using the uniform mesh, choose $h_{0}=\frac{\sqrt{2}}{8}, h_{1}=\frac{\sqrt{2}}{32}, h_{2}=\frac{\sqrt{2}}{64}$, and $h_{3}=\frac{\sqrt{2}}{128}$; then we have $h_{i}=h_{i-1}^{t_{i}}$, i.e., 
$\delta_{h_{i}}\left(\lambda_{k}\right)=\delta_{h_{i}-1}^{t_{i}}\left(\lambda_{k}\right)$, where $t_{1} \approx 1.80, t_{2} \approx 1.22, t_{3} \approx 1$.18. For a nonsmooth eigenfunction, the condition could be met when the local refinement is done near the singular point.

Condition 4.3 For any given number $\beta_{0} \in(0,1)$, there exists $0<\beta_{0} \leq \beta_{i}<1(i=1,2, \ldots)$ such that $\delta_{h_{i}}\left(\lambda_{k}\right)=\beta_{i} \delta_{h_{i-1}}\left(\lambda_{k}\right), \delta_{h_{i}}\left(\lambda_{k}\right) \rightarrow 0(i \rightarrow \infty)$.

Theorem 4.2 Let $\left(\lambda_{k}^{h_{l}}, u_{k}^{h_{l}}\right)$ be an approximate eigenpair obtained by Scheme 1. Suppose that Condition 4.2 holds, then there exist $u_{k} \in M\left(\lambda_{k}\right)$ and $H_{0}>0$ such that if $H<H_{0}$, Lemma 2.1 and the following estimates hold:

$$
\begin{aligned}
& \left\|u_{k}^{h_{l}}-u_{k}\right\|_{h} \leq C_{0} \delta_{h_{l}}\left(\lambda_{k}\right), \\
& \left|\lambda_{k}^{h_{l}}-\lambda_{k}\right| \leq C_{0}^{1+r} C_{7} \delta_{h_{l}}^{1+r}\left(\lambda_{k}\right) .
\end{aligned}
$$

Proof The proof is completed by using induction and Theorem 4.1 with $\lambda_{0}=\lambda_{k}^{h_{l-1}}$. Note that $\delta_{H}\left(\lambda_{k}\right) \rightarrow 0$, then there is a proper small $H_{0}>0$ such that if $H \leq H_{0}$, Lemma 2.1 and the following inequalities hold:

$$
\begin{aligned}
& C_{0} \delta_{H}\left(\lambda_{k}\right) \leq \delta_{0}, \quad C_{0}^{1+r} C_{7} \delta_{H}^{1+r}\left(\lambda_{k}\right) \leq \delta_{0}, \\
& C_{0}^{2+2 r} C_{7}^{2} \delta_{H}^{2 r}\left(\lambda_{k}\right)+C_{0}^{2+r} C_{7} \delta_{H}^{r}\left(\lambda_{k}\right) \leq 1 .
\end{aligned}
$$

When $l=1$, we have $\left(\lambda_{k}^{h_{l-1}}, u_{k}^{h_{l-1}}\right)=\left(\lambda_{k, H}, u_{k, H}\right)$; from Lemma 2.1 and (2.12), we know that there exists $\bar{u} \in M\left(\lambda_{k}\right)$ such that

$$
\begin{aligned}
& \left\|u_{k, H}-\bar{u}\right\|_{H} \leq C_{5} \delta_{H}\left(\lambda_{k}\right) \leq \delta_{0}, \\
& \left|\lambda_{k, H}-\lambda_{k}\right| \leq C_{5}^{1+r} C_{7} \delta_{H}^{1+r}\left(\lambda_{k}\right) \leq \delta_{0},
\end{aligned}
$$

and $\delta_{h_{1}}\left(\lambda_{j}\right) \leq \delta_{0}(j=k-1, k, k+q, j \neq 0)$, i.e. Condition 4.1 holds. Thus, by Theorem 4.1 and $2-t_{1} \geq 0$ and $C_{5} \leq C_{0}$ we get

$$
\begin{aligned}
\left\|u_{k}^{h_{1}}-u_{k}\right\|_{h} & \leq \frac{C_{0}}{2}\left\{C_{5}^{2+2 r} C_{7}^{2} \delta_{H}^{2+2 r}\left(\lambda_{k}\right)+C_{5}^{2+r} C_{7} \delta_{H}^{2+r}\left(\lambda_{k}\right)+\delta_{h_{1}}\left(\lambda_{k}\right)\right\} \\
& \leq \frac{C_{0}}{2}\left\{C_{0}^{2+2 r} C_{7}^{2} \delta_{H}^{2+2 r-t_{1}}\left(\lambda_{k}\right)+C_{0}^{2+r} C_{7} \delta_{H}^{2+r-t_{1}}\left(\lambda_{k}\right)+1\right\} \delta_{h_{1}}\left(\lambda_{k}\right) \\
& \leq \frac{C_{0}}{2}\left\{C_{0}^{2+2 r} C_{7}^{2} \delta_{H}^{2 r}\left(\lambda_{k}\right)+C_{0}^{2+r} C_{7} \delta_{H}^{r}\left(\lambda_{k}\right)+1\right\} \delta_{h_{1}}\left(\lambda_{k}\right) \\
& \leq C_{0} \delta_{h_{1}}\left(\lambda_{k}\right) .
\end{aligned}
$$

Combining (2.12) and the above inequality yields

$$
\left|\lambda_{k}^{h_{1}}-\lambda_{k}\right| \leq C_{7}\left\|u_{k}^{h_{1}}-u_{k}\right\|_{h}^{1+r} \leq C_{0}^{1+r} C_{7} \delta_{h_{1}}^{1+r}\left(\lambda_{k}\right) .
$$

Suppose that Theorem 4.2 is valid for $l-1$, i.e. there exists $\bar{u} \in M\left(\lambda_{k}\right)$ such that

$$
\begin{aligned}
& \left\|u_{k}^{h_{l-1}}-\bar{u}\right\|_{h} \leq C_{0} \delta_{h_{l-1}}\left(\lambda_{k}\right), \\
& \left|\lambda_{k}^{h_{l-1}}-\lambda_{k}\right| \leq C_{0}^{1+r} C_{7} \delta_{h_{l-1}}^{1+r}\left(\lambda_{k}\right),
\end{aligned}
$$


then, owing to (4.13)-(4.14), we have $\left\|u_{k}^{h_{l-1}}-\bar{u}\right\|_{h} \leq \delta_{0}$ and $\left|\lambda_{k}^{h_{l-1}}-\lambda_{k}\right| \leq \delta_{0}(j=k-1, k, k+$ $q, j \neq 0$ ), i.e. the conditions of Theorem 4.1 hold. Therefore, for $l$, by (4.6) and (4.14) we deduce

$$
\begin{aligned}
\left\|u_{k}^{h_{l}}-u_{k}\right\|_{h} & \leq \frac{C_{0}}{2}\left\{C_{0}^{2+2 r} C_{7}^{2} \delta_{h_{l-1}}^{2+2 r}\left(\lambda_{k}\right)+C_{0}^{2+r} C_{7} \delta_{h_{l-1}}^{2+r}\left(\lambda_{k}\right)+\delta_{h_{l}}\left(\lambda_{k}\right)\right\} \\
& \leq \frac{C_{0}}{2}\left\{C_{0}^{2+2 r} C_{7}^{2} \delta_{h_{l-1}+2 r-t_{l}}\left(\lambda_{k}\right)+C_{0}^{2+r} C_{7} \delta_{h_{l-1}}^{2+r-t_{l}}\left(\lambda_{k}\right)+1\right\} \delta_{h_{l}}\left(\lambda_{k}\right) \\
& \leq \frac{C_{0}}{2}\left\{C_{0}^{2+2 r} C_{7}^{2} \delta_{H}^{2+2 r-t_{l}}\left(\lambda_{k}\right)+C_{0}^{2+r} C_{7} \delta_{H}^{2+r-t_{l}}\left(\lambda_{k}\right)+1\right\} \delta_{h_{l}}\left(\lambda_{k}\right) \\
& \leq \frac{C_{0}}{2}\left\{C_{0}^{2+2 r} C_{7}^{2} \delta_{H}^{2 r}\left(\lambda_{k}\right)+C_{0}^{2+r} C_{7} \delta_{H}^{r}\left(\lambda_{k}\right)+1\right\} \delta_{h_{l}}\left(\lambda_{k}\right) \\
& \leq C_{0} \delta_{h_{l}}\left(\lambda_{k}\right) .
\end{aligned}
$$

By (2.12) and the above inequality we deduce

$$
\left|\lambda_{k}^{h_{l}}-\lambda_{k}\right| \leq C_{7}\left\|u_{k}^{h_{l}}-u_{k}\right\|_{h}^{1+r} \leq C_{0}^{1+r} C_{7} \delta_{h_{l}}^{1+r}\left(\lambda_{k}\right)
$$

i.e. (4.11)-(4.12) are valid.

Theorem 4.3 Let $\left(\lambda_{k}^{h_{l}}, u_{k}^{h_{l}}\right)$ be an approximate eigenpair obtained by Scheme 2. Suppose that Condition 4.2 holds for $i \leq i_{0}$ and Condition 4.3 holds for $i>i_{0}$. Then there exist $u_{k} \in$ $M\left(\lambda_{k}\right)$ and $H_{0}>0$ such that if $H \leq H_{0}$ it holds that

$$
\begin{aligned}
& \left\|u_{k}^{h_{l}}-u_{k}\right\|_{h} \leq C_{0} \delta_{h_{l}}\left(\lambda_{k}\right), \\
& \left|\lambda_{k}^{h_{l}}-\lambda_{k}\right| \leq C_{0}^{1+r} C_{7} \delta_{h_{l}}^{1+r}\left(\lambda_{k}\right), \quad l>i_{0} .
\end{aligned}
$$

Proof The proof is completed by using induction and Theorem 4.1 with $\lambda_{0}=\lambda_{k}^{h_{i 0}}$. Note that $\delta_{H}\left(\lambda_{k}\right) \rightarrow 0(H \rightarrow 0)$, then there is a proper small $H_{0}>0$ such that if $H \leq H_{0}$, Lemma 2.1 and the following inequalities hold:

$$
\begin{aligned}
& C_{0} \delta_{H}\left(\lambda_{k}\right) \leq \delta_{0}, \quad C_{0}^{1+r} C_{7} \delta_{H}^{1+r}\left(\lambda_{k}\right) \leq \delta_{0}, \\
& C_{0}^{2+2 r} C_{7}^{2} \delta_{h_{l_{0}+1}}^{1+r}\left(\lambda_{k}\right) \delta_{h_{l-1}}^{r}\left(\lambda_{k}\right) \frac{1}{\beta_{0}}+C_{0}^{2+r} C_{7} \delta_{h_{l_{0}+1}}^{1+r}\left(\lambda_{k}\right) \frac{1}{\beta_{0}} \leq 1 .
\end{aligned}
$$

When $l=i_{0}+1$, by Theorem 4.2 we know that there exists $u_{k} \in M\left(\lambda_{k}\right)$ such that

$$
\begin{aligned}
& \left\|u_{k}^{h_{i_{0}+1}}-u_{k}\right\|_{h} \leq C_{0} \delta_{h_{i_{0}+1}}\left(\lambda_{k}\right), \\
& \left|\lambda_{k}^{h_{i_{0}+1}}-\lambda_{k}\right| \leq C_{0}^{1+r} C_{7} \delta_{h_{i+1}}^{1+r}\left(\lambda_{k}\right) .
\end{aligned}
$$

Suppose that Theorem 4.3 holds for $l-1$, i.e. there exists $\bar{u} \in M\left(\lambda_{k}\right)$ such that

$$
\begin{aligned}
& \left\|u_{k}^{h_{l-1}}-\bar{u}\right\|_{h} \leq C_{0} \delta_{h_{l-1}}\left(\lambda_{k}\right), \\
& \left|\lambda_{k}^{h_{l-1}}-\lambda_{k}\right| \leq C_{0}^{1+r} C_{7} \delta_{h_{l-1}}^{1+r}\left(\lambda_{k}\right) .
\end{aligned}
$$


Then we infer from (4.17) that the conditions of Theorem 4.1 hold; therefore, for $l$, we can get

$$
\begin{aligned}
& \left\|u_{k}^{h_{l}}-u_{k}\right\|_{h} \\
& \quad \leq \frac{C_{0}}{2}\left\{C_{0}^{2+2 r} C_{7}^{2} \delta_{h_{l_{0}+1}}^{1+r}\left(\lambda_{k}\right) \delta_{h_{l-1}}^{1+r}\left(\lambda_{k}\right)+C_{0}^{2+r} C_{7} \delta_{h_{l_{0}+1}}^{1+r}\left(\lambda_{k}\right) \delta_{h_{l-1}}\left(\lambda_{k}\right)+\delta_{h_{l}}\left(\lambda_{k}\right)\right\} \\
& \quad \leq \frac{C_{0}}{2}\left\{C_{0}^{2+2 r} C_{7}^{2} \delta_{h_{l_{0}+1}}^{1+r}\left(\lambda_{k}\right) \delta_{h_{l-1}}^{r}\left(\lambda_{k}\right) \frac{1}{\beta_{0}}+C_{0}^{2+r} C_{7} \delta_{h_{l_{0}+1}}^{1+r}\left(\lambda_{k}\right) \frac{1}{\beta_{0}}+1\right\} \delta_{h_{l}}\left(\lambda_{k}\right),
\end{aligned}
$$

which together with (4.18), we get (4.15). Substituting (4.15) into the inequality (2.12), we get (4.16).

Remark For some adaptive local refined grids used usually, (2.9) can be expressed as $\left|E_{h}(u, v)\right| \leq C_{4} h\|v\|_{h}, \forall v \in S^{h}+H_{0}^{2}(\Omega)$, therefore $r$ in the theorems of this paper can take 1 .

\section{Adaptive algorithms}

In this section, referring to $[10,17,27]$, we present six algorithms. We denote Algorithm 1 in [10] as Algorithm 1 in this paper, and Algorithms 2-3 are established based on Schemes $1-2$, respectively. Then we combine Algorithms 1-3 with a uniformly refined algorithm to get Algorithms $1 \mathrm{M}-3 \mathrm{M}$, respectively. And the a posterior error estimator in the following algorithms comes from [4], that is

$$
\begin{aligned}
\eta_{h}\left(f, w_{h}, \kappa\right)^{2}= & h_{\kappa}^{4}\|f\|_{0, \kappa}^{2} \\
& +\sum_{F \in \varepsilon_{h} \cap \partial \kappa} h_{F}\left\|\frac{1}{2}\left[\left(\nabla\left(\nabla w_{h}\right)+\nabla\left(\nabla w_{h}\right)^{T}\right) \tau_{F}\right]\right\|_{0, F}^{2} \text { in } \mathbb{R}^{2}, \\
\eta_{h}\left(f, w_{h}, \kappa\right)^{2}= & h_{\kappa}^{4}\|f\|_{0, \kappa}^{2} \\
& +\sum_{F \in \varepsilon_{h} \cap \partial \kappa} h_{F}\left\|\frac{1}{2}\left[\left(\nabla\left(\nabla w_{h}\right)+\nabla\left(\nabla w_{h}\right)^{T}\right) \times \gamma_{F}\right]\right\|_{0, F}^{2} \text { in } \mathbb{R}^{3}, \\
\eta_{h}\left(f, w_{h}, \pi_{h}\right)^{2}= & \sum_{\kappa \in \pi_{h}} \eta_{h}\left(f, w_{h}, \kappa\right)^{2},
\end{aligned}
$$

where $w_{h}$ is the finite element approximate solution of $(2.4), \tau_{F}$ is the tangential vector and $\gamma_{F}$ the unit outward normal on $F \in \varepsilon_{h}$.

In the following algorithms, we have to provide an initial shape regular triangulation $\pi_{h_{0}}$ and a parameter $\theta \in(0,1)$. Also, from $[10,11]$ we know that replacing $w_{h}$ with $u_{h}$ and replacing $f$ with $\lambda_{h} u_{h}$ in (5.1), we can obtain the error estimator of Algorithms 1 and 1M. By Lemma 4.1 we can deduce that replacing $w_{h}$ with $u^{h}$ and replacing $f$ with $\lambda^{h} u^{h}$ in (5.1), we can obtain the error estimator of Algorithms 2-3 and Algorithms 2M-3M.

Algorithm 1 Choose the parameter $0<\theta<1$.

Step 1. Pick any initial mesh $\pi_{h_{0}}$.

Step 2. Solve (2.3) on $\pi_{h_{0}}$ for discrete solution $\left(\lambda_{h_{0}}, u_{h_{0}}\right)$.

Step $3 . l \Leftarrow 0$.

Step 4. Compute the local indicators $\eta_{h_{l}}\left(\lambda_{h_{l}} u_{h_{l}}, u_{h_{l}}, \kappa\right)$.

Step 5. Construct $\hat{\pi}_{h_{l}} \in \pi_{h_{l}}$ by Marking strategy E and $\theta$. 
Step 6 . Refine $\pi_{h_{l}}$ to get a new mesh $\pi_{h_{l+1}}$ by procedure Refine.

Step 7. Solve (2.3) on $\pi_{h_{l+1}}$ for discrete solution $\left(\lambda_{h_{l+1}}, u_{h_{l+1}}\right)$.

Step $8 . l \Leftarrow l+1$ and go to Step 4 .

Algorithm 2 Choose the parameter $0<\theta<1$.

Step 1. Pick any initial mesh $\pi_{h_{0}}$.

Step 2. Solve (2.3) on $\pi_{h_{0}}$ for discrete solution $\left(\lambda_{h_{0}}, u_{h_{0}}\right)$.

Step 3. $l \Leftarrow 0, \lambda_{0} \Leftarrow \lambda_{h_{0}}, u^{h_{0}} \Leftarrow u_{h_{0}}$.

Step 4. Compute the local indicators $\eta_{h_{l}}\left(\lambda^{h_{l}} u^{h_{l}}, u^{h_{l}}, \kappa\right)$.

Step 5. Construct $\hat{\pi}_{h_{l}} \in \pi_{h_{l}}$ by Marking strategy E1 and $\theta$.

Step 6. Refine $\pi_{h_{l}}$ to get a new mesh $\pi_{h_{l+1}}$ by procedure Refine.

Step 7. Find $u^{\prime} \in V_{h_{l+1}}$ such that

$$
a_{h}\left(u^{\prime}, v\right)-\lambda_{0} b\left(u^{\prime}, v\right)=b\left(u^{h_{l}}, v\right)
$$

denote $u^{h_{l+1}}=\frac{u^{\prime}}{\left\|u^{\prime}\right\|_{h}}$ and compute the Rayleigh quotient:

$$
\lambda^{h_{l+1}}=\frac{a_{h}\left(u^{h_{l+1}}, u^{h_{l+1}}\right)}{b\left(u^{h_{l+1}}, u^{h_{l+1}}\right)} .
$$

Step 8. $\lambda_{0} \Leftarrow \lambda^{h_{l+1}}, l \Leftarrow l+1$ and go to Step 4 .

Algorithm 3 Choose the parameter $0<\theta<1$ and an integer $i_{0}$.

Step 1 Step 7. The same as Steps 1-7 of Algorithm 2.

Step 8. If $l<i_{0}, \lambda_{0} \Leftarrow \lambda^{h_{l+1}}, l \Leftarrow l+1$ and go to Step 4; else $l \Leftarrow l+1$, and go to Step 4 .

A family of good adaptive meshes should satisfy $h=O\left(h_{\min }^{\alpha}\right)$. Hence, we give a bound $C_{r}$ of $\frac{h}{h_{\min }^{\alpha}}$. When the rate $\frac{h}{h_{\min }^{\alpha}} \geq C_{r}$ in the process of Algorithms $1 \mathrm{M}-3 \mathrm{M}$ is running, we refine the mesh uniformly for one time. And thus the following three algorithms are derived.

Algorithm 1M Choose the parameter $0<\theta<1, \alpha$, and a bound $C_{r}$ of $\frac{h_{l}}{h_{l_{\min }}^{\alpha}}$.

Step 1 Step 7. The same as Steps 1-7 of Algorithm 1.

Step $8 . l \Leftarrow l+1$.

Step 9. If $\frac{h_{l}}{h_{l_{\min }}} \geq C_{r}$, then uniformly refine the mesh $\pi_{h_{l}}$ to get a new mesh $\pi_{h_{l+1}}$ and go to Step 7, else go to Step 4.

Algorithm 2M Choose the parameter $0<\theta<1, \alpha$, and a bound $C_{r}$ of $\frac{h_{l}}{h_{l_{\min }}^{\alpha}}$.

Step 1 Step 7. The same as Steps 1-7 of Algorithm 2.

Step 8. $\lambda_{0} \Leftarrow \lambda^{h_{l+1}}, l \Leftarrow l+1$.

Step 9. If $\frac{h_{l}}{h_{l_{\min }}^{\alpha}} \geq C_{r}$, then uniformly refine the mesh $\pi_{h_{l}}$ to get a new mesh $\pi_{h_{l+1}}$ and go to Step 7, else go to Step 4.

Algorithm 3M Choose the parameter $0<\theta<1$, an integer $i_{0}, \alpha$, and a bound $C_{r}$ of $\frac{h_{l}}{h_{l_{\min }}^{\alpha}}$. Step 1 Step 7. The same as Steps 1-7 of Algorithm 2.

Step 8. If $l<i_{0}, \lambda_{0} \Leftarrow \lambda^{h_{l+1}}, l \Leftarrow l+1$; else $l \Leftarrow l+1$.

Step 9. If $\frac{h_{l}}{h_{l_{\min }}^{\alpha}} \geq C_{r}$, then uniformly refine the mesh $\pi_{h_{l}}$ to get a new mesh $\pi_{h_{l+1}}$ and go to Step 7, else go to Step 4. 
Marking strategy E Given parameter $0<\theta<1$ :

Step 1. Construct a minimal subset $\widehat{\pi}_{h_{l}}$ of $\pi_{h_{l}}$ by selecting some elements in $\pi_{h_{l}}$ such that

$$
\sum_{\kappa \in \widehat{\pi}_{h_{l}}} \eta_{h_{l}}^{2}\left(\lambda_{h_{l}} u_{h_{l}}, u_{h_{l}}, \kappa\right) \geq \theta \eta_{h_{l}}^{2}\left(\lambda_{h_{l}} u_{h_{l}}, u_{h_{l}}, \Omega\right)
$$

Step 2. Mark all the elements in $\widehat{\pi}_{h_{l}}$.

Marking strategy E1 To get Marking strategy E1 we only replace $\lambda_{h_{l}}$ and $u_{h_{l}}$ in Marking strategy E with $\lambda^{h_{l}}$ and $u^{h_{l}}$, respectively.

Algorithms 1M-3M including steps with uniform refinement seem to be opposite to the adaptive concept. Indeed, the combination of adaptive algorithms and uniform refinement meets the certain mesh-grading properties, thus improving the efficiency of Algorithms 1-3 (see Tables 1-3 in Sect. 6).

\section{Numerical experiment}

In this section, we compute the smallest eigenvalue of (2.1) on the L-shaped domain $(0,1)^{2} \backslash\left[\frac{1}{2}, 1\right]^{2}$ by Algorithms $1-3$ and Algorithms $1 \mathrm{M}-3 \mathrm{M}$ and $(0,1)^{3} \backslash([0.5,1] \times[0,1] \times$ $[0.5,1])$ by Algorithms $1-2$ to demonstrate the advantages of the adaptive Morley element method based on the inverse-shift iteration for a biharmonic eigenvalue problem. Our programs are compiled on MATLAB2012a under the package of Chen [28] using HP-Z230 workstation with ROM 32G and CPU $3.60 \mathrm{GHz}$.

We use the command " $\backslash$ " to solve (5.2) and use the sparse solver eigs $\left(A, B, 1,{ }^{\prime} \mathrm{sm}^{\prime}\right)$ to solve (2.3) for the smallest eigenvalues. Before showing the results, some symbols need to be explained:

$\lambda_{h_{l}}$ the smallest eigenvalue obtained by the $l$ th iteration using Algorithm 1.

$\lambda_{h_{l}}^{R}$ the smallest eigenvalue obtained by the $l$ th iteration using Algorithm 2.

$\lambda_{h_{l}}^{F}$ the smallest eigenvalue obtained by the $l$ th iteration using Algorithm 3.

$\lambda_{h_{l}}^{M}$ the smallest eigenvalue obtained by the $l$ th iteration using Algorithm $1 \mathrm{M}$.

$\lambda_{h_{l}}^{R M}$ the smallest eigenvalue obtained by the $l$ th iteration using Algorithm 2M.

$\lambda_{h_{l}}^{F M}$ the smallest eigenvalue obtained by the $l$ th iteration using Algorithm 3M.

$N_{\text {dof }}$ the number of the degree of freedom.

$\mathrm{CPU}(s)$ the time CPU runs from the first iteration to the current iteration.

In $\mathbb{R}^{2}$, the initial mesh $\pi_{h_{0}}$ is isosceles right triangle subdivision with mesh size $\frac{\sqrt{2}}{32}$, and we take $\theta=0.25, C_{r}=1.1, \alpha=\frac{1}{2}$. We fix shift from the 25th and 13th in Algorithm 3 and Algorithm 3M, respectively. The results are shown in Tables $1-3$. We depict the error curves of Algorithms 1-3 and Algorithms 1M-3M in Figs. 1-3.

From Tables 1-3, we can get the conclusion that in the case the accurate are almost same, Algorithms 2-3 take about half time of Algorithm 1. In the case the accurate are almost same, Algorithm $i \mathrm{M}$ takes about $\frac{2}{5}$ time of Algorithm $i, i=1,2,3$.

The smallest eigenvalue of (2.1) is unknown. Therefore, we replace it with an approximate eigenvalue $\lambda_{1} \approx 6703.585$ in $\mathbb{R}^{2}$ with high accuracy. It is present that the relative error curves of the smallest eigenvalues derived from Algorithms 1-3 and Algorithms 1M-3M on the adaptive meshes in Figs. 1-3, whose slopes are more or less -1 , which shows that all the six Morley element adaptive algorithms can get the optimal convergence rate $O\left(h^{2}\right)$ in $\mathbb{R}^{2}$. 
Table 1 The smallest eigenvalue solved by Algorithm 1 and Algorithm 1M

\begin{tabular}{|c|c|c|c|c|c|c|c|c|c|c|}
\hline I & $N_{\text {dof }}$ & $h_{1}$ & $\frac{h_{1}}{h_{1}^{\alpha}}$ & $\lambda_{1, h_{1}}$ & CPU(s) & $N_{\text {dof }}$ & $h_{1}$ & $\frac{h_{1}}{h_{I_{\text {min }}^{\alpha}}^{\alpha}}$ & $\lambda_{1, h_{l}}^{M}$ & CPU(s) \\
\hline 1 & 2945 & 0.044 & 0.210 & 6333.637 & 0.275 & 2945 & 0.044 & 0.210 & 6333.637 & 0.086 \\
\hline 2 & 2957 & 0.044 & 0.250 & 6368.756 & 0.368 & 2957 & 0.044 & 0.250 & 6368.756 & 0.162 \\
\hline 3 & 3035 & 0.044 & 0.297 & 6426.312 & 0.452 & 3035 & 0.044 & 0.297 & 6426.312 & 0.239 \\
\hline 4 & 3135 & 0.044 & 0.354 & 6459.396 & 0.537 & 3135 & 0.044 & 0.354 & 6459.396 & 0.320 \\
\hline 5 & 3345 & 0.044 & 0.420 & 6506.181 & 0.629 & 3345 & 0.044 & 0.420 & 6506.181 & 0.405 \\
\hline 6 & 3609 & 0.044 & 0.500 & 6540.464 & 0.726 & 3609 & 0.044 & 0.500 & 6540.464 & 0.499 \\
\hline 7 & 3979 & 0.044 & 0.595 & 6574.027 & 0.834 & 3979 & 0.044 & 0.595 & 6574.027 & 0.605 \\
\hline 8 & 4459 & 0.044 & 0.707 & 6588.671 & 0.957 & 4459 & 0.044 & 0.707 & 6588.671 & 0.723 \\
\hline 9 & 5097 & 0.044 & 0.841 & 6606.244 & 1.10 & 5097 & 0.044 & 0.841 & 6606.244 & 0.860 \\
\hline 10 & 5787 & 0.044 & 1.00 & 6615.776 & 1.27 & 5787 & 0.044 & 1.00 & 6615.776 & 1.02 \\
\hline 11 & 6665 & 0.044 & 1.19 & 6631.992 & 1.48 & 6665 & 0.044 & 1.19 & 6631.992 & 1.21 \\
\hline 12 & 7791 & 0.044 & 1.41 & 6642.939 & 1.71 & 31,697 & 0.022 & 0.841 & 6688.240 & 2.12 \\
\hline 13 & 9110 & 0.044 & 1.68 & 6656.854 & 1.97 & 34,833 & 0.022 & 1.00 & 6690.595 & 3.15 \\
\hline 14 & 10,591 & 0.044 & 2.00 & 6662.468 & 2.27 & 39,191 & 0.022 & 1.19 & 6693.066 & 4.43 \\
\hline 15 & 12,295 & 0.044 & 2.38 & 6665.980 & 2.65 & 173,919 & 0.011 & 0.841 & 6701.061 & 11.4 \\
\hline 16 & 14,331 & 0.044 & 2.83 & 6671.824 & 3.10 & 189,989 & 0.011 & 1.00 & 6701.477 & 19.4 \\
\hline 17 & 16,641 & 0.044 & 3.36 & 6676.967 & 3.62 & 211,977 & 0.011 & 1.19 & 6701.750 & 29.0 \\
\hline 18 & 19,497 & 0.044 & 4.00 & 6680.844 & 4.21 & 948,969 & 0.006 & 0.841 & 6703.147 & 82.8 \\
\hline 19 & 22,925 & 0.044 & 4.76 & 6684.502 & 4.92 & $1,025,149$ & 0.006 & 1.00 & 6703.198 & 141 \\
\hline 20 & 27,171 & 0.044 & 5.66 & 6686.546 & 5.77 & $1,131,177$ & 0.006 & 1.19 & 6703.244 & 210 \\
\hline 21 & 32,088 & 0.044 & 6.73 & 6689.797 & 6.76 & $5,114,697$ & 0.003 & 0.841 & 6703.512 & 587 \\
\hline 22 & 37,703 & 0.044 & 8.00 & 6692.349 & 7.95 & - & - & - & - & - \\
\hline 23 & 44,289 & 0.044 & 9.51 & 6694.425 & 9.39 & - & - & - & - & - \\
\hline 24 & 52,103 & 0.044 & 11.3 & 6695.960 & 11.1 & - & - & - & - & - \\
\hline 25 & 60,857 & 0.044 & 13.5 & 6696.560 & 13.2 & - & - & - & - & - \\
\hline 26 & 70,881 & 0.044 & 16.0 & 6697.400 & 15.7 & - & - & - & - & - \\
\hline 27 & 83,091 & 0.031 & 13.5 & 6698.304 & 18.7 & - & - & - & - & - \\
\hline 28 & 98,019 & 0.031 & 16.0 & 6699.274 & 22.7 & - & - & - & - & - \\
\hline 29 & 116,273 & 0.031 & 19.0 & 6699.950 & 27.4 & - & - & - & - & - \\
\hline 30 & 136,557 & 0.031 & 22.6 & 6700.589 & 33.1 & - & - & - & - & - \\
\hline 31 & 160,465 & 0.022 & 16.0 & 6701.087 & 40.0 & - & - & - & - & - \\
\hline 32 & 188,195 & 0.022 & 19.0 & 6701.469 & 48.2 & - & - & - & - & - \\
\hline 33 & 221,401 & 0.022 & 22.6 & 6701.858 & 58.0 & - & - & - & - & - \\
\hline 34 & 257,797 & 0.022 & 26.9 & 6702.060 & 69.7 & - & - & - & - & - \\
\hline 35 & 301,063 & 0.022 & 32.0 & 6702.246 & 84.6 & - & - & - & - & - \\
\hline 36 & 353,201 & 0.022 & 38.1 & 6702.411 & 102 & - & - & - & - & - \\
\hline 37 & 416,609 & 0.022 & 45.3 & 6702.557 & 124 & - & - & - & - & - \\
\hline 38 & 492,039 & 0.022 & 45.3 & 6702.767 & 151 & - & - & - & - & - \\
\hline 39 & 577,233 & 0.022 & 53.8 & 6702.937 & 182 & - & - & - & - & - \\
\hline 40 & 677,271 & 0.016 & 45.3 & 6703.035 & 220 & - & - & - & - & - \\
\hline 41 & 793,765 & 0.016 & 53.8 & 6703.115 & 266 & - & - & - & - & - \\
\hline 42 & 934,557 & 0.016 & 64.0 & 6703.190 & 321 & - & - & - & - & - \\
\hline 43 & $1,084,193$ & 0.016 & 64.0 & 6703.237 & 388 & - & - & - & - & - \\
\hline 44 & $1,267,059$ & 0.016 & 76.1 & 6703.272 & 465 & - & - & - & - & - \\
\hline 45 & $1,487,051$ & 0.016 & 90.5 & 6703.320 & 558 & - & - & - & - & - \\
\hline 46 & $1,756,709$ & 0.016 & 108 & 6703.362 & 672 & - & - & - & - & - \\
\hline 47 & $2,065,245$ & 0.011 & 90.5 & 6703.407 & 809 & - & - & - & - & - \\
\hline 48 & $2,420,223$ & 0.011 & 108 & 6703.446 & 973 & - & - & - & - & - \\
\hline 49 & $2,834,373$ & 0.011 & 128 & 6703.468 & 1171 & - & - & - & - & - \\
\hline 50 & $3,319,763$ & 0.011 & 128 & 6703.487 & 1415 & - & - & - & - & - \\
\hline 51 & $3,894,763$ & 0.011 & 152 & 6703.505 & 1706 & - & - & - & - & - \\
\hline 52 & $4,522,239$ & 0.011 & 181 & 6703.516 & 2060 & - & - & - & - & - \\
\hline
\end{tabular}

In $\mathbb{R}^{3}$, the initial mesh $\pi_{h_{0}}$ is tetrahedron subdivision with mesh size $\frac{\sqrt{3}}{16}$, and we take $\theta=$ 0.25 and $\lambda_{1} \approx 8290.011$ with high accuracy replacing the accurate eigenvalue. It is present that the refined mesh and the relative error curves of the smallest eigenvalues derived from Algorithms 1-2 in Fig. 4, from which we see that Algorithm 2 is more efficient than Algorithm 1, but meanwhile we also see from Table 4 that the mesh size has no change. 
Table 2 The smallest eigenvalue solved by Algorithm 2 and Algorithm 2M

\begin{tabular}{|c|c|c|c|c|c|c|c|c|c|c|}
\hline I & $N_{\text {dof }}$ & $h_{1}$ & $\frac{h_{1}}{h_{\text {min }}^{\alpha}}$ & $\lambda_{1, h_{l}}^{R}$ & $\mathrm{CPU}(\mathrm{s})$ & $N_{\text {dof }}$ & $h_{1}$ & $\frac{h_{1}}{h_{\text {min }}^{\alpha}}$ & $\lambda_{1, h_{l}}^{\mathrm{RM}}$ & $\mathrm{CPU}(s)$ \\
\hline 1 & 2945 & 0.044 & 0.210 & 6333.637 & 0.133 & 2945 & 0.044 & 0.210 & 6333.637 & 0.090 \\
\hline 2 & 2957 & 0.044 & 0.297 & 6373.503 & 0.228 & 2957 & 0.044 & 0.297 & 6373.503 & 0.140 \\
\hline 3 & 3031 & 0.044 & 0.354 & 6538.971 & 0.280 & 3031 & 0.044 & 0.354 & 6538.971 & 0.192 \\
\hline 4 & 3067 & 0.044 & 0.420 & 6443.737 & 0.371 & 3067 & 0.044 & 0.420 & 6443.737 & 0.250 \\
\hline 5 & 3237 & 0.044 & 0.500 & 6489.761 & 0.426 & 3237 & 0.044 & 0.500 & 6489.761 & 0.305 \\
\hline 6 & 3445 & 0.044 & 0.595 & 6523.466 & 0.487 & 3445 & 0.044 & 0.595 & 6523.466 & 0.365 \\
\hline 7 & 3811 & 0.044 & 0.707 & 6566.620 & 0.560 & 3811 & 0.044 & 0.707 & 6566.620 & 0.431 \\
\hline 8 & 4195 & 0.044 & 0.841 & 6595.431 & 0.636 & 4195 & 0.044 & 0.841 & 6595.431 & 0.504 \\
\hline 9 & 4678 & 0.044 & 1.00 & 6597.955 & 0.720 & 4678 & 0.044 & 1.00 & 6597.955 & 0.588 \\
\hline 10 & 5293 & 0.044 & 1.19 & 6607.441 & 0.814 & 5293 & 0.044 & 1.19 & 6607.441 & 0.709 \\
\hline 11 & 6118 & 0.044 & 1.41 & 6623.248 & 0.924 & 25,297 & 0.022 & 0.841 & 6683.573 & 1.15 \\
\hline 12 & 6997 & 0.044 & 1.68 & 6634.588 & 1.05 & 27,723 & 0.022 & 1.00 & 6686.324 & 1.63 \\
\hline 13 & 8232 & 0.044 & 2.00 & 6648.804 & 1.20 & 30,933 & 0.022 & 1.19 & 6688.518 & 2.38 \\
\hline 14 & 9527 & 0.044 & 2.38 & 6658.106 & 1.39 & 139,409 & 0.011 & 0.841 & 6700.069 & 5.87 \\
\hline 15 & 11,102 & 0.044 & 2.83 & 6663.393 & 1.59 & 153,179 & 0.011 & 1.00 & 6700.762 & 9.71 \\
\hline 16 & 12,928 & 0.044 & 3.36 & 6667.166 & 1.82 & 168,897 & 0.011 & 1.19 & 6701.161 & 15.4 \\
\hline 17 & 15,139 & 0.044 & 4.00 & 6673.763 & 2.11 & 740,417 & 0.006 & 0.841 & 6702.983 & 39.1 \\
\hline 18 & 17,619 & 0.044 & 4.76 & 6678.433 & 2.43 & 807,451 & 0.006 & 1.00 & 6703.097 & 65.4 \\
\hline 19 & 20,763 & 0.044 & 5.66 & 6682.562 & 2.82 & 882,597 & 0.006 & 1.19 & 6703.149 & 103 \\
\hline 20 & 24,365 & 0.044 & 6.73 & 6685.164 & 3.27 & $3,907,351$ & 0.003 & 0.841 & 6703.486 & 236 \\
\hline 21 & 28,967 & 0.044 & 8.00 & 6687.944 & 3.81 & $4,218,771$ & 0.003 & 1.00 & 6703.504 & 382 \\
\hline 22 & 34,068 & 0.044 & 9.51 & 6690.675 & 4.50 & - & - & - & - & - \\
\hline 23 & 40,007 & 0.044 & 11.3 & 6692.914 & 5.39 & - & - & - & - & - \\
\hline 24 & 47,117 & 0.044 & 13.5 & 6694.937 & 6.46 & - & - & - & - & - \\
\hline 25 & 55,275 & 0.044 & 16.0 & 6696.294 & 7.64 & - & - & - & - & - \\
\hline 26 & 64,407 & 0.044 & 19.0 & 6696.867 & 9.09 & - & - & - & - & - \\
\hline 27 & 75,259 & 0.031 & 13.5 & 6697.823 & 10.8 & - & - & - & - & - \\
\hline 28 & 88,353 & 0.031 & 16.0 & 6698.752 & 13.2 & - & - & - & - & - \\
\hline 29 & 104,269 & 0.031 & 19.0 & 6699.457 & 16.1 & - & - & - & - & - \\
\hline 30 & 123,285 & 0.031 & 22.6 & 6700.133 & 19.4 & - & - & - & - & - \\
\hline 31 & 145,061 & 0.031 & 26.9 & 6700.774 & 23.3 & - & - & - & - & - \\
\hline 32 & 170,397 & 0.022 & 22.6 & 6701.291 & 27.9 & - & - & - & - & - \\
\hline 33 & 199,833 & 0.022 & 26.9 & 6701.638 & 33.5 & - & - & - & - & - \\
\hline 34 & 235,261 & 0.022 & 26.9 & 6701.906 & 40.0 & - & - & - & - & - \\
\hline 35 & 272,877 & 0.022 & 32.0 & 6702.117 & 48.6 & - & - & - & - & - \\
\hline 36 & 319,549 & 0.022 & 38.1 & 6702.292 & 58.8 & - & - & - & - & - \\
\hline 37 & 375,279 & 0.022 & 45.3 & 6702.462 & 71.1 & - & - & - & - & - \\
\hline 38 & 444,149 & 0.022 & 53.8 & 6702.631 & 86.1 & - & - & - & - & - \\
\hline 39 & 522,525 & 0.022 & 64.0 & 6702.819 & 104 & - & - & - & - & - \\
\hline 40 & 613,375 & 0.022 & 76.1 & 6702.964 & 125 & - & - & - & - & - \\
\hline 41 & 719,217 & 0.016 & 53.8 & 6703.062 & 149 & - & - & - & - & - \\
\hline 42 & 844,333 & 0.016 & 64.0 & 6703.144 & 179 & - & - & - & - & - \\
\hline 43 & 988,863 & 0.016 & 76.1 & 6703.208 & 214 & - & - & - & - & - \\
\hline 44 & $1,150,057$ & 0.016 & 90.5 & 6703.256 & 255 & - & - & - & - & - \\
\hline 45 & $1,346,861$ & 0.016 & 108 & 6703.292 & 304 & - & - & - & - & - \\
\hline 46 & $1,584,041$ & 0.016 & 128 & 6703.337 & 362 & - & - & - & - & - \\
\hline 47 & $1,873,597$ & 0.016 & 152 & 6703.378 & 433 & - & - & - & - & - \\
\hline 48 & $2,196,067$ & 0.011 & 108 & 6703.422 & 509 & - & - & - & - & - \\
\hline 49 & $2,572,281$ & 0.011 & 128 & 6703.454 & 598 & - & - & - & - & - \\
\hline 50 & $3,010,543$ & 0.011 & 152 & 6703.474 & 705 & - & - & - & - & - \\
\hline 51 & $3,538,161$ & 0.011 & 181 & 6703.497 & 831 & - & - & - & - & - \\
\hline 52 & $4,120,833$ & 0.011 & 215 & 6703.510 & 979 & - & - & - & - & - \\
\hline
\end{tabular}


Table 3 The smallest eigenvalue solved by Algorithm 3 and Algorithm 3M

\begin{tabular}{|c|c|c|c|c|c|c|c|c|c|c|}
\hline I & $N_{\text {dof }}$ & $h_{1}$ & $\frac{h_{1}}{h_{I_{\text {min }}^{\alpha}}}$ & $\lambda_{1, h_{l}}^{F}$ & $\mathrm{CPU}(\mathrm{s})$ & $N_{\text {dof }}$ & $h_{1}$ & $\frac{h_{1}}{h_{I_{\text {min }}^{\alpha}}}$ & $\lambda_{1, h_{1}}^{F M}$ & $\mathrm{CPU}(s)$ \\
\hline 1 & 2945 & 0.044 & 0.210 & 6333.637 & 0.086 & 2945 & 0.044 & 0.210 & 6333.637 & 0.086 \\
\hline 2 & 2957 & 0.044 & 0.297 & 6373.503 & 0.137 & 2957 & 0.044 & 0.297 & 6373.503 & 0.137 \\
\hline 3 & 3031 & 0.044 & 0.354 & 6538.971 & 0.187 & 3031 & 0.044 & 0.354 & 6538.971 & 0.187 \\
\hline 4 & 3067 & 0.044 & 0.420 & 6443.737 & 0.244 & 3067 & 0.044 & 0.420 & 6443.737 & 0.245 \\
\hline 5 & 3237 & 0.044 & 0.500 & 6489.761 & 0.299 & 3237 & 0.044 & 0.500 & 6489.761 & 0.300 \\
\hline 6 & 3445 & 0.044 & 0.595 & 6523.466 & 0.358 & 3445 & 0.044 & 0.595 & 6523.466 & 0.360 \\
\hline 7 & 3811 & 0.044 & 0.707 & 6566.620 & 0.423 & 3811 & 0.044 & 0.707 & 6566.620 & 0.426 \\
\hline 8 & 4195 & 0.044 & 0.841 & 6595.431 & 0.496 & 4195 & 0.044 & 0.841 & 6595.431 & 0.498 \\
\hline 9 & 4678 & 0.044 & 1.00 & 6597.955 & 0.577 & 4678 & 0.044 & 1.00 & 6597.955 & 0.581 \\
\hline 10 & 5293 & 0.044 & 1.19 & 6607.441 & 0.670 & 5293 & 0.044 & 1.19 & 6607.441 & 0.696 \\
\hline 11 & 6118 & 0.044 & 1.41 & 6623.248 & 0.779 & 25,297 & 0.022 & 0.841 & 6683.573 & 1.14 \\
\hline 12 & 6997 & 0.044 & 1.68 & 6634.588 & 0.903 & 27,723 & 0.022 & 1.00 & 6686.324 & 1.62 \\
\hline 13 & 8232 & 0.044 & 2.00 & 6648.804 & 1.05 & 30,933 & 0.022 & 1.19 & 6688.518 & 2.37 \\
\hline 14 & 9527 & 0.044 & 2.38 & 6658.106 & 1.21 & 139,409 & 0.011 & 0.841 & 6700.069 & 5.85 \\
\hline 15 & 11,102 & 0.044 & 2.83 & 6663.393 & 1.41 & 153,179 & 0.011 & 1.00 & 6701.996 & 9.71 \\
\hline 16 & 12,928 & 0.044 & 3.36 & 6667.166 & 1.64 & 164,253 & 0.011 & 1.19 & 6701.274 & 15.2 \\
\hline 17 & 15,139 & 0.044 & 4.00 & 6673.763 & 1.93 & 715,319 & 0.006 & 0.841 & 6702.994 & 38.2 \\
\hline 18 & 17,619 & 0.044 & 4.76 & 6678.433 & 2.25 & 780,735 & 0.006 & 1.00 & 6703.096 & 63.8 \\
\hline 19 & 20,763 & 0.044 & 5.66 & 6682.562 & 2.63 & 853,934 & 0.006 & 1.19 & 6703.205 & 100 \\
\hline 20 & 24,365 & 0.044 & 6.73 & 6685.164 & 3.08 & $3,774,935$ & 0.003 & 0.841 & 6703.503 & 231 \\
\hline 21 & 28,967 & 0.044 & 8.00 & 6687.944 & 3.60 & $4,083,915$ & 0.003 & 1.00 & 6703.538 & 372 \\
\hline 22 & 34,068 & 0.044 & 9.51 & 6690.675 & 4.21 & - & - & - & - & - \\
\hline 23 & 40,007 & 0.044 & 11.3 & 6692.914 & 4.97 & - & - & - & - & - \\
\hline 24 & 47,117 & 0.044 & 13.5 & 6694.937 & 5.89 & - & - & - & - & - \\
\hline 25 & 55,275 & 0.044 & 16.0 & 6696.294 & 6.98 & - & - & - & - & - \\
\hline 26 & 64,407 & 0.044 & 19.0 & 6696.867 & 8.28 & - & - & - & - & - \\
\hline 27 & 75,259 & 0.031 & 13.5 & 6697.823 & 9.85 & - & - & - & - & - \\
\hline 28 & 88,357 & 0.031 & 16.0 & 6698.753 & 12.0 & - & - & - & - & - \\
\hline 29 & 104,277 & 0.031 & 19.0 & 6699.461 & 14.6 & - & - & - & - & - \\
\hline 30 & 123,275 & 0.031 & 22.6 & 6700.132 & 17.7 & - & - & - & - & - \\
\hline 31 & 145,073 & 0.031 & 26.9 & 6700.784 & 21.4 & - & - & - & - & - \\
\hline 32 & 170,409 & 0.022 & 22.6 & 6701.303 & 25.9 & - & - & - & - & - \\
\hline 33 & 199,844 & 0.022 & 26.9 & 6701.644 & 31.2 & - & - & - & - & - \\
\hline 34 & 235,273 & 0.022 & 26.9 & 6701.901 & 37.6 & - & - & - & - & - \\
\hline 35 & 272,825 & 0.022 & 32.0 & 6702.117 & 45.7 & - & - & - & - & - \\
\hline 36 & 319,389 & 0.022 & 38.1 & 6702.299 & 55.5 & - & - & - & - & - \\
\hline 37 & 375,188 & 0.022 & 45.3 & 6702.459 & 67.4 & - & - & - & - & - \\
\hline 38 & 443,902 & 0.022 & 53.8 & 6702.642 & 81.7 & - & - & - & - & - \\
\hline 39 & 522,189 & 0.022 & 64.0 & 6702.815 & 98.8 & - & - & - & - & - \\
\hline 40 & 612,931 & 0.022 & 76.1 & 6702.966 & 119 & - & - & - & - & - \\
\hline 41 & 718,761 & 0.016 & 53.8 & 6703.061 & 143 & - & - & - & - & - \\
\hline 42 & 844,127 & 0.016 & 64.0 & 6703.150 & 171 & - & - & - & - & - \\
\hline 43 & 988,405 & 0.016 & 76.1 & 6703.208 & 205 & - & - & - & - & - \\
\hline 44 & $1,149,526$ & 0.016 & 90.5 & 6703.256 & 244 & - & - & - & - & - \\
\hline 45 & $1,346,037$ & 0.016 & 108 & 6703.295 & 291 & - & - & - & - & - \\
\hline 46 & $1,583,069$ & 0.016 & 128 & 6703.340 & 347 & - & - & - & - & - \\
\hline 47 & $1,872,353$ & 0.016 & 152 & 6703.381 & 412 & - & - & - & - & - \\
\hline 48 & $2,194,659$ & 0.011 & 108 & 6703.425 & 490 & - & - & - & - & - \\
\hline 49 & $2,570,539$ & 0.011 & 128 & 6703.458 & 580 & - & - & - & - & - \\
\hline 50 & $3,008,669$ & 0.011 & 152 & 6703.478 & 687 & - & - & - & - & - \\
\hline 51 & $3,535,715$ & 0.011 & 181 & 6703.498 & 813 & - & - & - & - & - \\
\hline 52 & $4,118,331$ & 0.011 & 215 & 6703.512 & 962 & - & - & - & - & - \\
\hline
\end{tabular}



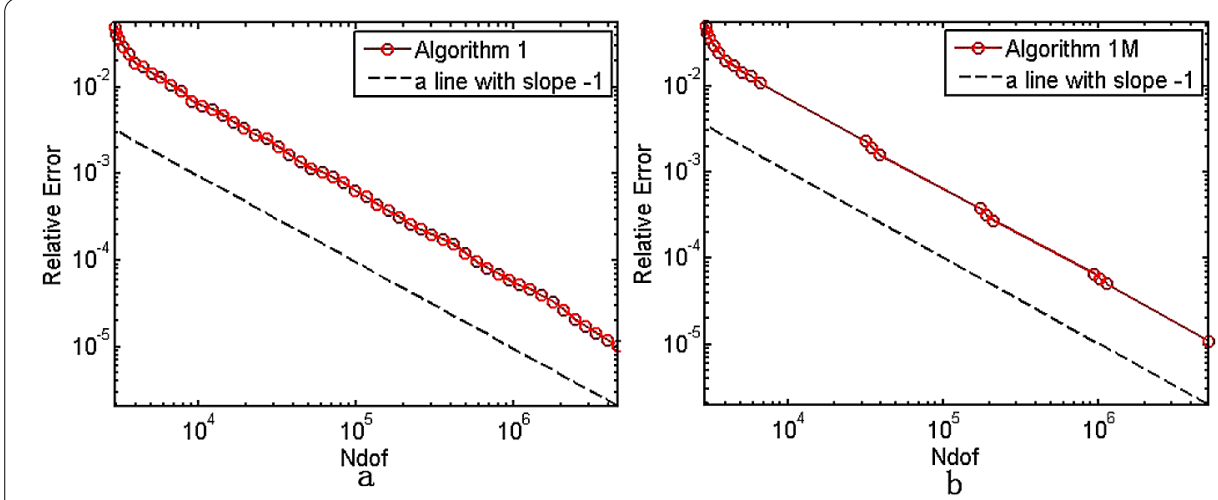

Figure 1 The convergence rates of the smallest eigenvalue from Algorithm 1(a) and Algorithm 1M(b)
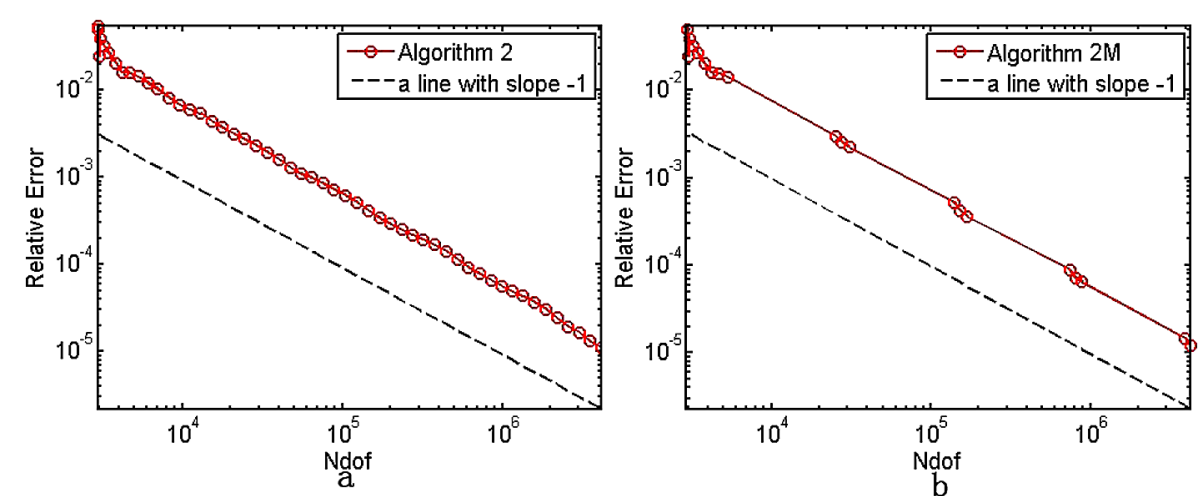

Figure 2 The convergence rates of the smallest eigenvalue from Algorithm 2(a) and Algorithm 2M(b)
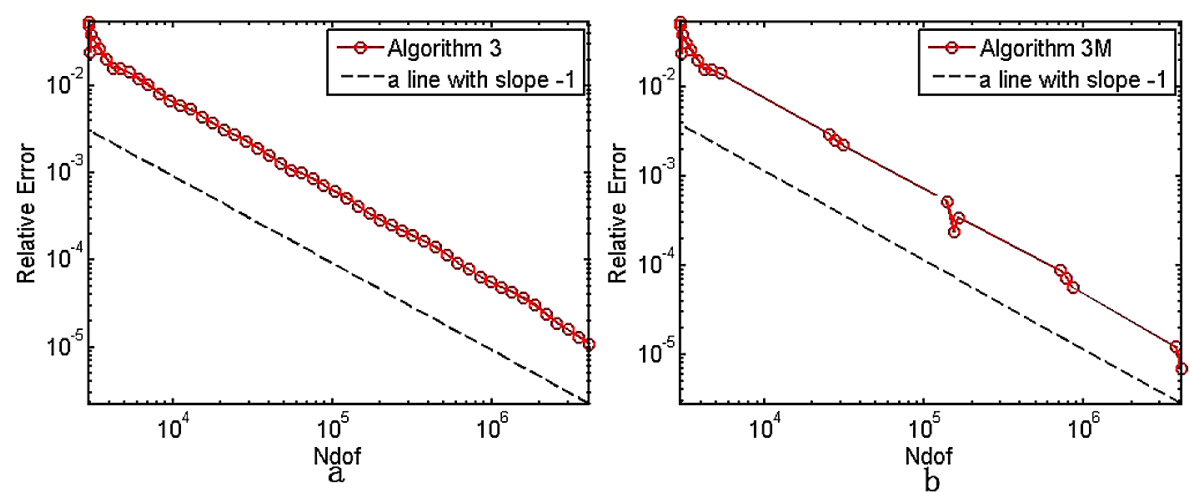

Figure 3 The convergence rates of the smallest eigenvalue from Algorithm 3(a) and Algorithm 3M(b) 


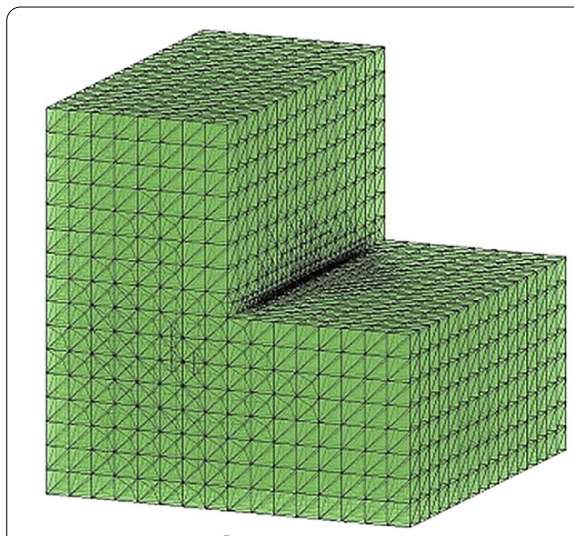

a

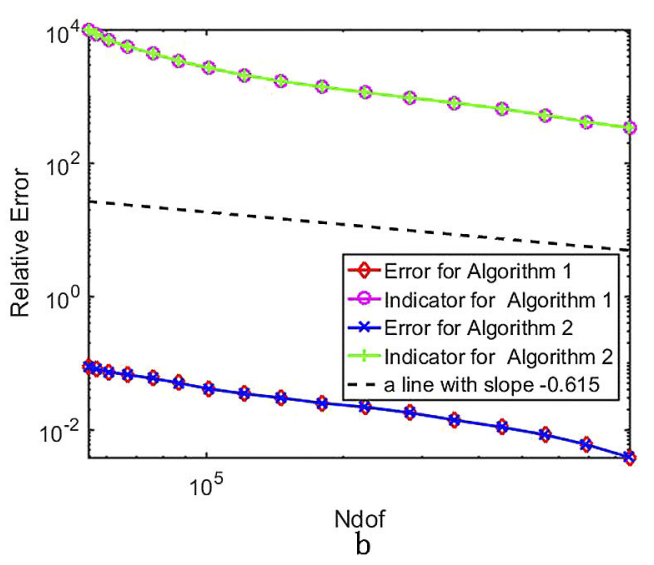

Figure 4 The refined mesh for the L-shaped domain (a) and the convergence rates of the smallest eigenvalue from Algorithm 1 and Algorithm 2(b) in $\mathbb{R}^{3}$

Table 4 The smallest eigenvalue solved by Algorithm 1 and Algorithm 2

\begin{tabular}{rrlllrlrr}
\hline$l$ & $N_{\text {dof }}$ & $h_{l}$ & \multicolumn{1}{l}{$\lambda_{1, h_{l}}$} & \multicolumn{1}{c}{ CPU(s) } & \multicolumn{1}{c}{$N_{\text {dof }}$} & $h_{l}$ & $\lambda_{1, h_{l}}^{R}$ & CPU(s) \\
\hline 1 & 54,896 & 0.108 & 7547.686 & 8.25 & 54,896 & 0.108 & 7547.686 & 9.71 \\
2 & 57,176 & 0.108 & 7612.980 & 16.5 & 57,176 & 0.108 & 7613.142 & 13.0 \\
3 & 60,822 & 0.108 & 7678.863 & 25.6 & 60,822 & 0.108 & 7678.902 & 16.7 \\
4 & 67,192 & 0.108 & 7736.644 & 35.1 & 67,192 & 0.108 & 7736.699 & 20.9 \\
5 & 76,278 & 0.108 & 7802.128 & 46.5 & 76,316 & 0.108 & 7802.062 & 25.8 \\
6 & 86,838 & 0.108 & 7871.038 & 58.6 & 86,905 & 0.108 & 7872.137 & 31.2 \\
7 & 101,261 & 0.108 & 7949.987 & 73.8 & 101,368 & 0.108 & 7950.349 & 38.4 \\
8 & 121,408 & 0.108 & 8001.974 & 92.4 & 121,563 & 0.108 & 8002.931 & 47.3 \\
9 & 146,456 & 0.108 & 8041.421 & 118 & 146,215 & 0.108 & 8040.738 & 58.1 \\
10 & 180,528 & 0.108 & 8082.211 & 155 & 180,203 & 0.108 & 8082.247 & 72.3 \\
11 & 224,755 & 0.108 & 8108.734 & 211 & 224,288 & 0.108 & 8108.530 & 92.1 \\
12 & 282,583 & 0.108 & 8141.177 & 295 & 281,953 & 0.108 & 8141.009 & 119 \\
13 & 355,133 & 0.108 & 8174.424 & 414 & 354,598 & 0.108 & 8174.437 & 156 \\
14 & 451,162 & 0.108 & 8199.573 & 583 & 450,739 & 0.108 & 8199.502 & 205 \\
15 & 561,904 & 0.108 & 8220.566 & 847 & 561,090 & 0.108 & 8220.433 & 269 \\
16 & 693,222 & 0.108 & 8240.310 & 2368 & 691,963 & 0.108 & 8240.129 & 354 \\
17 & 863,420 & 0.108 & 8258.156 & 9957 & 861,795 & 0.108 & 8258.042 & 469 \\
18 & - & - & - & - & $1,084,848$ & 0.108 & 8272.888 & 624 \\
19 & - & - & - & - & $1,357,830$ & 0.108 & 8281.060 & 851 \\
20 & - & - & - & - & $1,730,050$ & 0.108 & 8290.011 & 1206 \\
\hline
\end{tabular}

Because $N_{\text {dof }}$ in $\mathbb{R}^{3}$ increases very fast after uniform refinement, which leads to surpassing computer's memory, we cannot employ Algorithms $1 \mathrm{M}-3 \mathrm{M}$ to solve (2.1).

\section{Acknowledgements}

This work is supported by the Science and Technology Foundation of Guizhou Province of China (Grant nos. LH [2014] 7061 and LKS [2013] 06). We appreciate editors and reviewers for constructive suggestions and helpful comments.

\section{Competing interests}

The authors declare to have no competing interests.

\section{Authors' contributions}

$\mathrm{HL}$ and $Y Y$ participated in the theoretical analysis and $\mathrm{HL}$ carried out the numerical experiments. The final manuscript was made after YY had discussed it with HL. All authors read and approved the final manuscript.

\section{Publisher's Note}

Springer Nature remains neutral with regard to jurisdictional claims in published maps and institutional affiliations. 
Received: 8 December 2017 Accepted: 20 February 2018 Published online: 06 March 2018

\section{References}

1. Morley, L.S.D.: The triangular equilibrium element in the solution of plate bending problems. Aero Quart (1968)

2. Wang, M., Xu, J.: The Morley element for fourth order elliptic equations in any dimensions 155-169. Numer. Math. 103, $155-169(2006)$

3. da Veiga, L.B., Niiranen, J., Stenberg, R.: A posteriori errors estimates for the Morley plate bending element. Numer. Math. 106, 165-179 (2007)

4. Hu, J., Shi, Z.: A new a posteriori error estimate for the Morley element. Numer. Math. 112, 25-40 (2009)

5. Shi, Z., Wang, M.: Finite Element Methods. Science Press, Beijing (2013)

6. Carstensen, C., Gallistl, D., Hu, J.: A posteriori error estimates for nonconforming finite element methods for fourth-order problems on rectangles. Numer. Math. 124, 309-335 (2013)

7. Hu, J., Shi, Z., Xu, J.: Convergence and optimality of the adaptive Morley method. Numer. Math. 121, $731-752$ (2012)

8. Carstensen, C., Gallistl, D., Hu, J.: A discrete Helmholtz decomposition with Morley finite element functions and the optimality of adaptive finite element schemes. Comput. Math. Appl. 68(part B), 2167-2181 (2014)

9. Rannacher, R.: Nonconforming finite element methods for eigenvalue problems in linear plate theory. Numer. Math. 13, 23-42 (1979)

10. Shen, Q.: A posteriori error estimates of the Morley element for the fourth order elliptic eigenvalue problem. Numer. Algorithms 68, 455-466 (2015)

11. Yang, Y., Li, H., Bi, H.: The lower bound property of the Morley element eigenvalues. Comput. Math. Appl. 17, 904-920 (2016)

12. Gallistl, D.: Morley finite element method for the eigenvalues of the biharmonic operator. IMA J. Numer. Anal. 35(4), 1779-1811 (2015)

13. Babuška, I., Rheinboldt, W.C.: Error estimates for adaptive finite element computations. SIAM J. Numer. Anal. 15 736-754 (1978)

14. Verfürth, R.: A Review of a Posteriori Error Estimates and Adaptive Mesh-Refinement Techniques. Wiley-Teubner, New York (1996)

15. Ainsworth, M., Oden, J.T.: A Posteriori Error Estimates in the Finite Element Analysis. Wiley-Inter science, New York (2011)

16. Morin, P., Nochetto, R.H., Siebert, K.: Convergence of adaptive finite element methods. SIAM Rev. 44, 631-658 (2002)

17. Yang, Y., Bi, H., Han, J., Yu, Y.: The shifted-inverse iteration based on the multigrid discretizations for eigenvalue problems. SIAM J. Sci. Comput. 37, 2583-2606 (2015)

18. Blum, H., Rannacher, R.: On the boundary value problem of the biharmonic operator on domains with angular corners. Math. Methods Appl. Sci. 2, 556-581 (1980)

19. Li, H., Yang, Y.: $c^{0}$ ipg adaptive algorithms for the biharmonic eigenvalue problem. Numer. Algorithms (2017). https://doi.org/10.1007/s11075-017-0388-8

20. Ciarlet, P.G.: Basic Error Estimates for Elliptic Problems. Handbook of Numerical Analysis, vol. 2. Elsevier, North-Holand (1991)

21. Babuška, I., Kellog, R.B., Pitkaranta, J.: Direct and inverse error estimates for finite elements with mesh refinement. Numer. Math. 33, 447-471 (1979)

22. Hu, J., Huang, Y., Lin, Q.: Lower bounds for eigenvalues of elliptic operators: by nonconforming finite element methods. J. Sci. Comput. 61, 196-221 (2014)

23. Lin, Q., Xie, H., Xu, J.: Lower bounds of the discretization for piecewise polynomials. Math. Comput. 83, 1-13 (2014)

24. Carstensen, C., Gallistl, D.: Guaranteed lower eigenvalue bounds for the biharmonic equation. Numer. Math. 125, 33-51 (2014)

25. Yang, Y., Zhang, Z., Lin, F.: Eigenvalue approximation from below using nonforming finite elements. Sci. China Math. 53, 137-150 (2010)

26. Yang, Y., Bi, H.: A tow-grid discretization scheme based on shifted-inverse power method. SIAM J. Numer. Anal. 49 $1602-1642(2011)$

27. Dai, X., Xu, J., Zhou, A.: Convergence and optimal complexity of adaptive finite element eigenvalue computations. Numer. Math. 110, 313-355 (2008)

28. Chen, L. (ed.): iFEM: An Innovative Finite Element Methods Package in MATLAB. Technical Report, University of California at Irvine (2009)

\section{Submit your manuscript to a SpringerOpen ${ }^{\circ}$ journal and benefit from:}

- Convenient online submission

- Rigorous peer review

- Open access: articles freely available online

- High visibility within the field

- Retaining the copyright to your article

Submit your next manuscript at $>$ springeropen.com 